Ergod. Th. \& Dynam. Sys. (1982), 2, 317-337

Printed in Great Britain

\title{
Invariant measures for some one-dimensional attractors
}

\author{
M. V. J ACOBSON
}

Moscow, USSR

(Received 10 March 1982)

Dedicated to the memory of V. M. Alexeyev to whom I shall always be grateful for his friendship and encouragement

Abstract. We consider certain non-invertible maps of the square which are extensions of the quadratic maps of the interval and their small perturbations. We show that several maps of the type possess attractors which are not hyperbolic but have invariant measures similar to Bowen-Ruelle measures for hyperbolic attractors.

1. The properties of a dynamical system $f: X \rightarrow X$ which possesses an invariant contracting foliation are closely related to the properties of the system induced on the quotient space. When $(X, f)$ is studied from the point of view of the topology, this relation is based on the inverse limit construction (see [14]), but when $f$-invariant measures are studied one uses the natural extension construction (see [12]).

In this paper we consider the situation when the quotient space is an interval and the corresponding induced one-dimensional map has a single critical point. We shall see that several systems of this type possess attractors which are not hyperbolic but have similar properties.

The maps under consideration are non-invertible. We show, however, that the set where the inverse map is not defined is negligible from the point of view of any absolutely continuous measure. We shall say that an attractor $\Lambda$ of the map $f$ admits an absolutely continuous invariant Bernoulli measure if there exists a finite measure $\mu$ such that $(\Lambda, f, \mu)$ is a Bernoulli automorphism and there is an invariant subset $\Lambda_{1} \subset \Lambda$ satisfying $\mu\left(\Lambda_{1}\right)=1$, where $\Lambda_{1}$ is a union of rectifiable curves $\gamma_{\alpha}$, forming $(\bmod 0)$ a measurable partition of $\Lambda_{1}$, and on every curve $\gamma_{\alpha} \mu$ induces the conditional measure $\mu\left(\cdot \mid \gamma_{\alpha}\right)$ which is absolutely continuous with respect to normalized length.

In $\$ \S 2-5$ we consider a map $F: S \rightarrow S$ of the unit square $S$ similar to the 'twisted horseshoe' map from [7] and prove the following theorem.

THEOREM 1. The attractor of the map $F$ admits an absolutely continuous Bernoulli measure. 
In $\$ \S 6-10$ we consider some one-parameter families of maps $F_{a}: S \rightarrow S$ which induce on the quotient space mappings $x \rightarrow g_{a}(x)$ close to $x \rightarrow a x(1-x)$. For these families we prove the following theorem using the results of [8].

THEOREM 2. There exists a set $M$ of parameter values of positive measure, so that for any $a \in M$ the attractor of the map $F_{a}$ admits an absolutely continuous Bernoulli measure.

The approach to the proof of these results is based on the 'induced map' method combined with the 'itinerary schemes' method due to Alexeyev [1].

In the last year of his life, despite his grave illness, V. M. Alexeyev continued his work on various mathematical problems. Several times we discussed the subject of this paper. His attentive and friendly attitude stimulated my work to a great extent.

I would like to thank B. M. Gurevich and I. P. Gornfeld for discussions and useful remarks.

2. We denote by $\mathscr{A} \subset C^{2}([0,1],[0,2])$ the set of mappings satisfying $g(0)=g(1)=0$. Let $\varepsilon>0$ be a small constant. We define $\mathscr{A}_{\varepsilon}<\mathscr{A}$ as the set of mappings $g$ satisfying

(i) $\|g(x)-4 x(1-x)\|_{c^{2}}<\varepsilon$;

(ii) $\max _{x \in[0,1]} g(x)=1$.

$\mathscr{A}_{\varepsilon}$ is a surface of codimension 1 in $\mathscr{A}$. The curve $\Gamma=\{x \rightarrow a x(1-x)\}$ intersects $\mathscr{A}_{\varepsilon}$ transversally at $a=4$, thus for any one-parameter family $x \rightarrow g_{a}(x) \in \mathscr{A}$ sufficiently close in $C^{2}(A \times[0,1],[0,2])$ to $x \rightarrow a x(1-x)$ where $a \in A=[0,5], x \in[0,1]$, there exists an $a_{0}$ such that $g_{a_{0}} \in \mathscr{A}_{\varepsilon}$.

Let us consider the square $S=\{0 \leq x \leq 1,0 \leq y \leq 1\}$ and the map $F: S \rightarrow S$ defined by

$$
F:(x, y) \rightarrow\left(\varphi(y)+\lambda\left(x-\frac{1}{2}\right), g(y)\right)
$$

where $\lambda>0$ is a small constant, $g(y) \in \mathscr{A}_{\varepsilon}$ and $\varphi(y)$ is a $C^{2}$ function satisfying the following conditions:

$$
\varphi(y)= \begin{cases}\varphi^{\prime}(y) \leq 1 \\ \frac{1}{4}+2 \delta & \text { for } y \in\left[0, \frac{1}{4}+\delta\right] \\ y & \text { for } y \in\left[r_{1}-\delta, 1-r_{1}+\delta\right] \\ \frac{3}{4}-2 \delta & \text { for } y \in\left[\frac{3}{4}-\delta, 1\right]\end{cases}
$$

where $r_{1}$ is defined in (3) and $\delta>0$ is the small constant defined in (4), see $\$ 3$.

The map $F$ is illustrated by figure 1 .

Remark. We have chosen the above formula for $\varphi$ in order to simplify the calculations. One can check that the following results are valid for a large class of $\varphi$.

A similar 'twisted horseshoe' map was analysed in [7]. Bowen in [4] considered such a map as an example whose dynamics are undecided. 


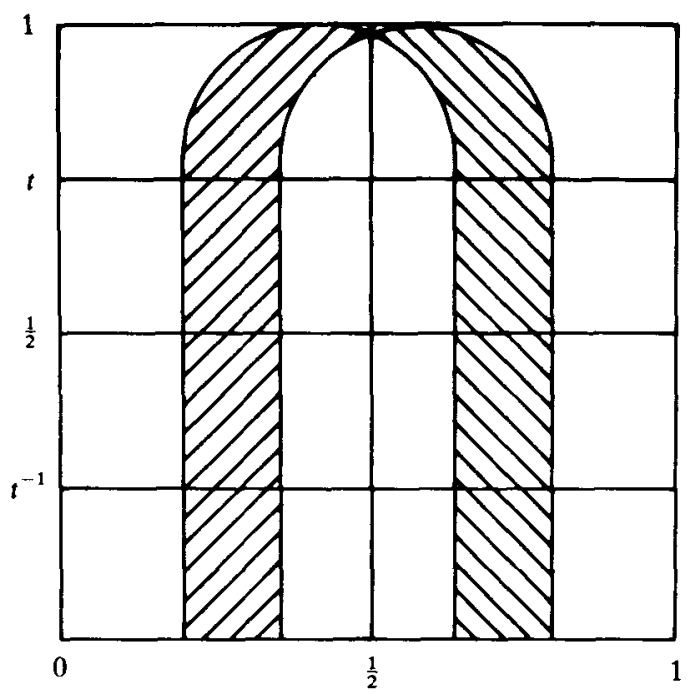

FIGURE 1

(1) implies that the horizontal foliation $\xi=\left\{\xi_{y}\right\}$ of $S$ is invariant under $F$, and $F: \xi_{y} \rightarrow \xi_{g(y)}$ is a contraction with coefficient $\lambda$.

Let us consider a sequence $\left(y_{0}, y_{1}, \ldots, y_{n}, \ldots\right)$, where $y_{n} \in g^{-1}\left(y_{n-1}\right)$, and $g^{-1}(y)$ is the inverse image of $y$ under $g$. We have $F^{n} \xi_{y_{n}} \subset \xi_{y}$, diam $F^{n} \xi_{y_{n}}<\lambda^{n}$ and thus $\bigcap_{n=0}^{\infty} F^{n} \xi_{y_{n}}$ is a point which we denote by $M\left(y_{0}, y_{1}, \ldots, y_{n}, \ldots\right)$.

Following the inverse limit construction (see [14]), we introduce a space $\tilde{Y}=$ $\left\{\tilde{y}=\left(y_{0}, y_{1}, \ldots\right)\right.$ and we define the map $\tilde{g}: \tilde{Y} \rightarrow \tilde{Y}$ by $\tilde{g}(\tilde{y})=\left(g\left(y_{0}\right), y_{0}, y_{1}, \ldots\right)$. We shall use the notation $\Lambda=\bigcap_{n=0}^{\infty} F^{n} S$, and $\pi:\left(y_{0}, y_{1}, \ldots\right) \rightarrow M\left(y_{0}, y_{1}, \ldots\right)$. It follows from the definition of the topology in $\tilde{Y}$ that $\pi$ is continuous, and the construction implies that $\pi: \tilde{Y} \rightarrow \Lambda$ is onto and satisfies

$$
\pi \circ \tilde{g}=F \circ \pi \text {. }
$$

3. The map $\pi$ is not one-to-one because $\tilde{g}$ is a homeomorphism and $F$ is noninvertible on $\Lambda$. We shall see nevertheless that $\pi$ induces an isomorphism between the dynamical system $\left(\Lambda, F, \mu_{*}\right)$ and the natural extension $(\tilde{Y}, \tilde{g}, \tilde{\mu})$ of the system $(Y, g, \mu)$, where $\mu$ is a $g$-invariant measure absolutely continuous with respect to the Lebesgue measure $d y$ on $[0,1]$, and $\mu_{*}=\pi_{*} \tilde{\mu}$.

It is known that any $g \in \mathscr{A}_{\varepsilon}$ admits an invariant measure $\mu_{\mathrm{g}}$ absolutely continuous with respect to $d y$. Let us recall the construction of $\mu_{\mathrm{g}}$ from [9].

The mapping $g$ has a repelling fixed point $t=g(t) \neq 0$. Let $t^{-1}$ be the second pre-image of $t$. Let us denote $I=\left[t^{-1}, t\right]$. Let $G: I \rightarrow I$ be the map induced by $g$, i.e., for $y \in I$ one sets $G(y)=g^{n(y)}(y)$, where

$$
n(y)=\min \left\{n \geq 1: g^{n}(y) \in I\right\} .
$$

We have $I=\left(\bigcup_{i=2}^{\infty} \Delta_{i \pm}\right) \cup K$, where $\Delta_{i \pm}$ are open intervals such that for

$$
\begin{gathered}
y \in \Delta_{i \pm} n(y)=i, \quad \Delta_{i-} \subset[0, c], \quad \Delta_{i+} \subset[c, 1], \\
g \Delta_{i+}=g \Delta_{i-}, \quad \Delta_{i} \cap \Delta_{j}=\varnothing,
\end{gathered}
$$


and $K$ is the union of the set of end points of $\Delta_{i \pm}$ and of the critical point $c$, see figure 2. Let us denote $g_{0}(y)=4 y(1-y)$. Then

$$
\mu_{0}=\mu_{\mathrm{g}_{0}}=d y / \pi \sqrt{y(1-y)}
$$

is the corresponding measure. For $g=g_{0}$ we have $I=I_{0}=\left[t_{0}, t_{0}^{-1}\right]$, where

$$
t_{0}=\frac{3}{4}, \quad t_{0}^{-1}=\frac{1}{4}, \quad \Delta_{2-}=\left[\frac{1}{4}, r_{1}\right], \quad \Delta_{2+}=\left[1-r_{1}, \frac{3}{4}\right) .
$$

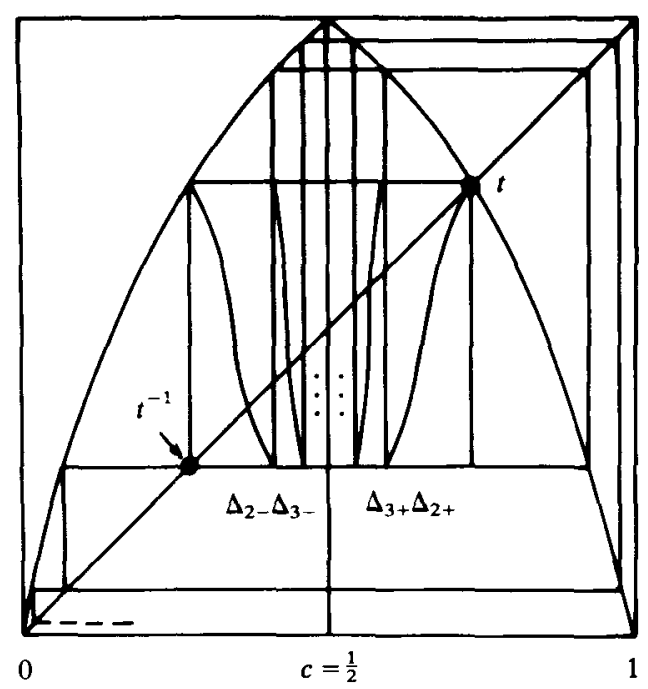

FIGURE 2

The map $G_{0}$ induced by $g_{0}$ satisfies the following conditions:

(A) $\exists k_{0}:\left|D G_{0}^{k_{0}}(y)\right|>c_{0}>1$ for any $y$ such that $G_{0}^{k_{0}}(y)$ is defined.

(B) $\sup _{y \in \Delta_{i}}\left|D^{2} G_{0}(y)\right| /\left|D G_{0}(y)\right| \cdot\left|\Delta_{i}\right|<c_{0}^{\prime}$, with $c_{0}^{\prime}$ independent from $i$.

If $\varepsilon$ is sufficiently small any $g \in \mathscr{A}_{\varepsilon}$ induces $G$ which also satisfies (A) and (B) on the interval $I=I_{g}=\left[t^{-1}, t\right]$. Let us denote

$$
\Delta_{2-}(g)=\left[t^{-1}, \rho_{1}\right], \quad \Delta_{2+}(g)=\left[\rho_{2}, t\right],
$$

and let $\delta$ be defined by

$$
\sup _{\mathbf{g} \in \mathscr{A}_{e}} \max \left\{\left|t^{-1}-\frac{1}{4}\right|,\left|\rho_{1}-r_{1}\right|,\left|\rho_{2}-\left(1-r_{1}\right)\right|,\left|t-\frac{3}{4}\right|\right\}=\delta / 2 .
$$

Here $\delta$ is the constant from the definition of $\varphi(y)$ in $\S 2$.

Conditions (A) and (B) imply ([2], [13]) that $G$ admits an invariant measure

$$
\nu_{8}(d y)=\chi(y) d y
$$

on $I$ with $\chi(y)$ continuous and bounded away from 0 .

The $f$-invariant measure $\mu_{\mathrm{g}}$ is constructed from $\nu_{\mathrm{g}}$ according to the following formula, which holds not only for the induced map $G$ but also in a more general 
situation when $G$ locally coincides with different powers of the map $g$ :

$$
\mu(E)=\sum_{i=1}^{\infty} \sum_{k=0}^{i-1} \nu\left(g^{-n}\left(E \cap g^{n} \Delta_{i}\right)\right)
$$

When $G$ is an induced map, we have $\mu \equiv \nu \mid I$ and $\mu(d y)=q(y) d y$ where the density $q(y)$ is continuous on $[0,1]$, bounded away from zero and has two singularities of the type $1 / \sqrt{y}$ at 0 and at 1 .

Let $(\tilde{I}, \tilde{G}, \tilde{\nu})$ be the natural extention of $(I, G, \nu)$. The construction of $\S 8$ below implies that the automorphisms of measure spaces $(\tilde{Y}, \tilde{g}, \tilde{\mu})$ and $(\tilde{I}, \tilde{G}, \tilde{\nu})$ are related in the following way. $(\tilde{I}, \tilde{G}, \tilde{\nu})$ is isomorphic to an automorphism induced by $\tilde{g}$ on a subset $\tilde{I}^{\prime}$ of $\tilde{Y}$. Let $\tilde{q}: \tilde{I} \rightarrow \tilde{I}^{\prime}$ be this isomorphism. For

$$
\tilde{z} \in \tilde{I}, \quad \tilde{z}=\left(z_{0}, z_{1}, z_{2}, \ldots\right), \quad G z_{i}=z_{i-1},
$$

for any $i=1,2, \ldots$ we have $G z_{i}=g^{n_{i}} z_{i}$. Then $\tilde{q}(\tilde{z})=\tilde{y}$ is defined by

$$
\tilde{y}=\left(z_{0}, g^{n_{1}-1} z_{1}, \ldots, g z_{1}, z_{1}, g^{n_{2}-1} z_{2}, \ldots, g z_{2}, z_{2}, \ldots\right) \text {. }
$$

Furthermore $\tilde{I}^{\prime}$ coincides mod 0 (i.e., neglecting sets of zero $\tilde{\mu}$-measure) with the set $\left\{\tilde{y}=\left(y_{0}, y_{1}, \ldots\right): y_{0} \in I\right\}$.

$(\tilde{I}, \tilde{G}, \tilde{v})$ admits a pair of invariant continuous partitions analogous to contracting and expanding foliations for Anosov systems (see [11, 12]). Let $\eta$ be the decreasing partition, whose elements are

$$
\eta_{J}=\left(I, \Delta_{j_{1}}, \Delta_{j_{2} j_{1}}, \ldots \Delta_{j_{n} \cdots j_{2} j_{1}}, \ldots\right)
$$

where $G \Delta_{j_{n} j_{n-1} \cdots j_{1}}=\Delta_{j_{n-1} \cdots j_{1}} ; J$ is used to denote the entire sequence $\left(j_{1}, j_{2}, \ldots\right) ; I$ is as above, and

$$
\Delta_{j_{n} j_{n-1} \cdots j_{1}}=\Delta_{j_{n}} \cap G^{-1} \Delta_{j_{n-1}} \cap \cdots \cap G^{-(n-1)} \Delta_{j_{1}} .
$$

Lemma 2 of $\$ 7$ implies that for any $\eta_{J}$ the conditional measure $\tilde{\nu}\left(\cdot \mid \eta_{J}\right)$ induced by $\tilde{\nu}$ on $\eta_{J}$ satisfies the following inequalities

$$
0<c_{2}<\tilde{\nu}\left(E \mid \eta_{J}\right) / \tilde{\nu}\left(\theta_{1} E\right)<c_{1}
$$

where $E$ is a measurable subset of $\eta_{J}, \theta_{1}: \tilde{I} \rightarrow I$ is the projection along the element of the increasing partition, and $c_{1}, c_{2}$ are independent from $J$.

When we consider the attractor $\Lambda$ the elements $\eta_{J}$ admit a natural geometric interpretation.

4. For a set $E \subset[0,1]$ on the $y$-axis we shall use the symbol $\Pi_{E}$ to denote the strip $\{(x, y): x \in[0,1], y \in E\}$.

Let $\tau$ be the map induced by $F$ on the strip $\Pi_{I}$ where $I=\left[t^{-1}, t\right]$ as in $\S 3$. The horizontal foliation $\xi=\left\{\xi_{y}\right\}$ is invariant under $\tau$ and we have

$$
\tau \xi_{y} \subset \xi_{G y} .
$$

The partition of $I$ into the intervals $\Delta_{i \pm}$ generates the partition of $\Pi_{I}$ into the strips $\Pi_{i \pm}$ (we use $\Pi_{i \pm}$ instead of $\Pi_{\Delta_{i \pm}}$ in order to simplify the notation). Let us consider the images $\tau \Pi_{i \pm}$. Figure 3 illustrates the map $F^{2}$ with the images $\tau \Pi_{2 \pm}=F^{2} \Pi_{2 \pm}$ shaded in. 


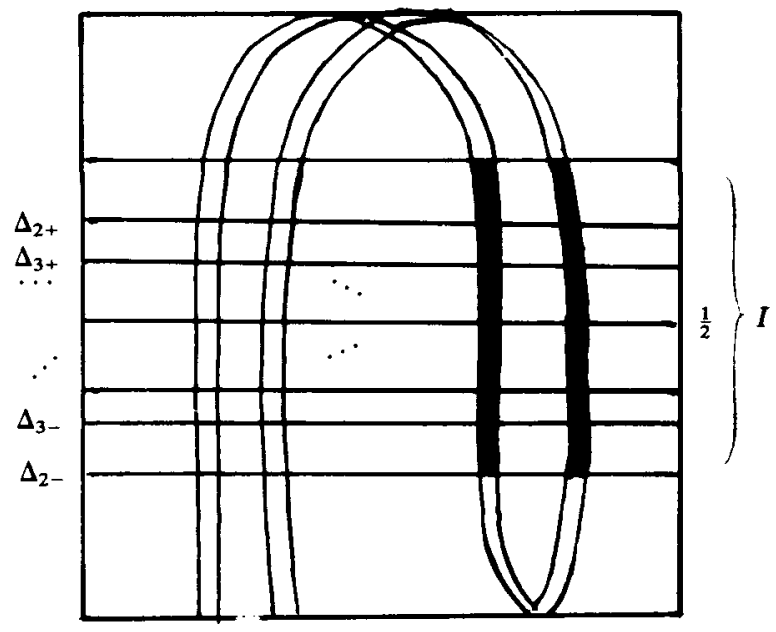

FIGURE 3

Let $a$ be the fixed point of the linear map

$$
x \rightarrow \frac{1}{4}+2 \nu+\lambda\left(x-\frac{1}{2}\right)
$$

The action of $F$ on $\Pi_{\left[0, t^{-1}\right]}$ may be written in the form

$$
(x-a, y) \rightarrow(\lambda(x-a), g(y)) .
$$

Thus $(a, 0)$ is a fixed saddle point of the map $F$, its stable manifold $W^{s}$ coincides with the $x$-axis, and the intersection of its unstable manifold $W^{u}$ with the strip $\Pi_{[0, t]}$ is a segment of the line $x=a$. As $F$ acts diffeomorphically on $\Pi_{\left[0, t^{-1}\right]}$, we see that the images $\tau \Pi_{n \pm}, \tau \Pi_{m \pm}$ do not intersect for $n \neq m$, that $\tau \Pi_{n+} \cap \tau \Pi_{n-}=\varnothing$ for small $n$ but $\tau \Pi_{n+} \cap \tau \Pi_{n-} \neq \varnothing$ for large $n$, and $\tau \Pi_{n}$ accumulate to $W^{u}$ when $n$ tends to infinity (see figure 4 ).

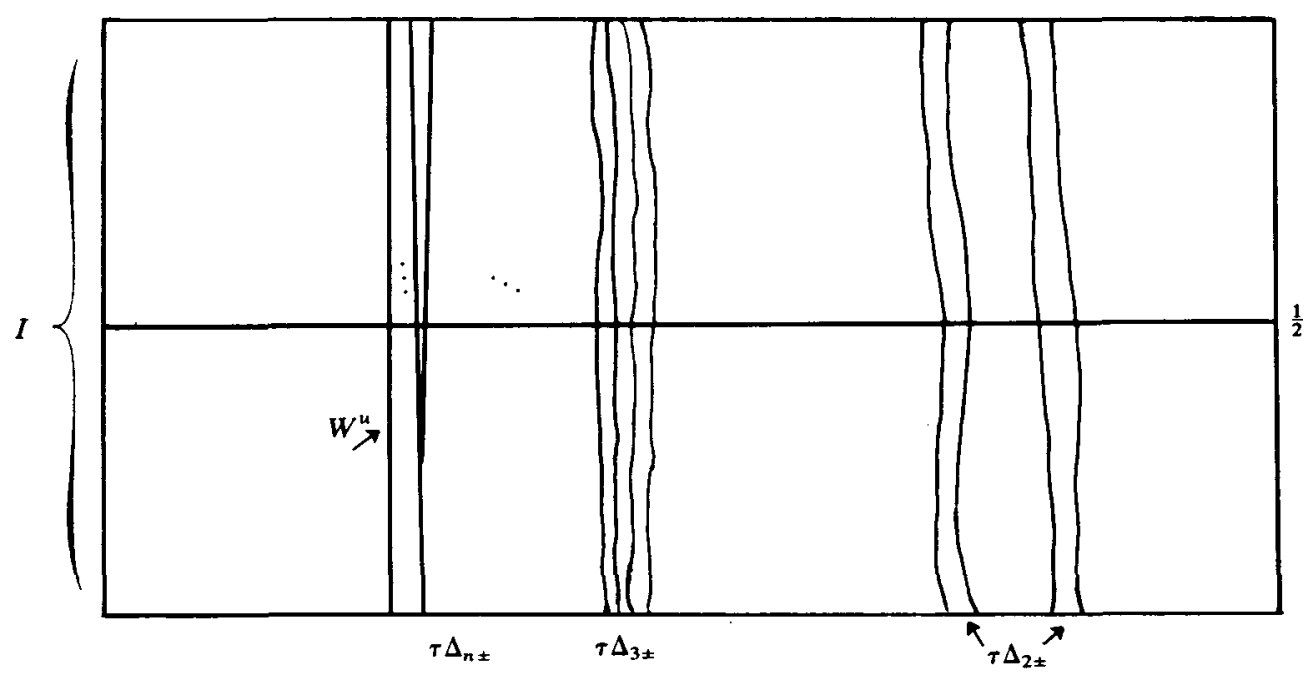

FIGURE 4 
We shall denote the map $\tau \mid \Pi_{n \pm}$ by $\tau_{n \pm}$. Let us consider the action of $D \tau_{n \pm}$. The derivative of $F$ equals

$$
D F(x, y)=\left(\begin{array}{ll}
\lambda & \varphi^{\prime}(y) \\
0 & g^{\prime}(y)
\end{array}\right) .
$$

The choice of $\delta$ in the definition of $\varphi(y)$ implies that all intervals $\Delta_{n \pm}$ with $n \geq 3$ are inside the domain where $\varphi^{\prime}(y) \equiv 1$, and besides

$$
\varphi^{\prime}(y) \equiv 0 \text { for } y \in\left[0, t^{-1}\right] \cup[t, 1] .
$$

Thus for $i \neq 2$ we have $\varphi^{\prime}(y)=1$, for any $y \in \Delta_{i}$, and for all $i=2,3, \ldots$ we have

$$
\varphi^{\prime}\left(g^{k} y\right)=0, k=1,2, \ldots, n_{i}-1 .
$$

Therefore we obtain for $n \geq 3$

$$
\begin{aligned}
D \tau_{n \pm}(x, y) & =\left(\begin{array}{cc}
\lambda^{n-1} & 0 \\
0 & \Pi_{k=1}^{n-1} g^{\prime}\left(g^{k} y\right)
\end{array}\right) \cdot\left(\begin{array}{cc}
\lambda & 1 \\
0 & g^{\prime}(y)
\end{array}\right) \\
& =\left(\begin{array}{cc}
\lambda^{n} & \lambda^{n-1} \\
0 & \left(g^{n}\right)^{\prime}(y)
\end{array}\right) .
\end{aligned}
$$

For $n=2$ we have

$$
\begin{aligned}
D \tau_{2 \pm}(x, y) & =\left(\begin{array}{cc}
\lambda & 0 \\
0 & g^{\prime}(g y)
\end{array}\right) \cdot\left(\begin{array}{ll}
\lambda & \varphi^{\prime}(y) \\
0 & g^{\prime}(y)
\end{array}\right) \\
& =\left(\begin{array}{cc}
\lambda^{2} & \lambda \varphi^{\prime}(y) \\
0 & \left(g^{2}\right)^{\prime}(y)
\end{array}\right) .
\end{aligned}
$$

Let $A=g^{\prime}(0)$ (for $g \in \mathscr{A}_{\varepsilon} A$ is close to 4). It is easy to check that there are constants $c_{3}, c_{4}>0$, such that for $n=2,3, \ldots$ and for any $y \in \Delta_{n \pm}$ the following inequality holds

$$
c_{3}(\sqrt{A})^{n}<\left|G^{\prime}(y)\right|=\left|\left(g^{n}\right)^{\prime}(y)\right|<c_{4}(\sqrt{A})^{n} .
$$

Let us consider the images $D \tau_{n} K$ of the cone

$$
K=\{(\xi, \eta):|\xi / \eta|<1\}
$$

in the tangent space TS. We have

$$
D \tau_{2}(\xi, \eta)=\left(\xi_{1}, \eta_{1}\right)=\left(\lambda^{2} \xi+\lambda \varphi_{(y)}^{\prime} \eta,\left(\left(g^{2}\right)^{\prime}(y)\right) \eta\right) .
$$

Thus

$$
\left|\xi_{1} / \eta_{1}\right|=\left|\frac{\lambda \varphi^{\prime}(y)}{\left(g^{2}\right)^{\prime}(y)}+\frac{\lambda^{2}}{\left(g^{2}\right)^{\prime}(y)} \frac{\xi}{\eta}\right|<\lambda \frac{1+\lambda}{A c_{3}} .
$$

For $n>2$ we have

$$
D \tau_{n}(\xi, \eta)=\left(\xi_{1}, \eta_{1}\right)=\left(\lambda^{n} \xi+\lambda^{n-1} \eta,\left(g^{n}\right)^{\prime}(y) \eta\right) .
$$

Thus

$$
\left|\xi_{1} / \eta_{1}\right|<\lambda^{n-1}\left((1+\lambda) /(\sqrt{A})^{n} \cdot c_{3}\right) .
$$

If $\lambda$ is sufficiently small the right-hand parts of (11) and (12) are less than 1 and we get $D \tau_{n \pm} K \subset K$ for all $n$. 
Let us denote $\Lambda^{\prime}=\cap_{p=0}^{\infty} \tau^{p} \Pi_{I}$ the attractor of the map $\tau: \Pi_{I} \rightarrow \Pi_{I}$. In order to study the structure of $\Lambda^{\prime}$ we use the 'itinerary schemes' method developed by Alexeyev [1]. In our situation Alexeyev's theorem (theorem $1^{-}$from [1]) may be formulated as follows.

Let us denote

$$
\begin{gathered}
D \tau_{n}=\left(\begin{array}{ll}
A_{11 n} & A_{12 n} \\
A_{21 n} & A_{22 n}
\end{array}\right) \\
\left\|A_{11 n}-A_{12 n} \cdot A_{22 n}^{-1} \cdot A_{21 n}\right\|=\mu_{1 n}, \quad\left\|A_{22 n}^{-1}\right\|=\mu_{2 n} \\
\left\|A_{12 n} \cdot A_{22 n}^{-1}\right\|=a_{1 n}, \quad\left\|A_{22 n}^{-1} \cdot A_{21 n}\right\|=a_{2 n} .
\end{gathered}
$$

Suppose the following conditions hold. There are two families of cones $K_{1}(x, y)$ and $K_{2}(x, y)$ in the tangent space so that

$$
K_{1} \ni(1,0), \quad K_{2} \ni(0,1), \quad K_{1} \cap K_{2}=\varnothing
$$

and for any $n$

(C) $\quad D \tau_{n} K_{1} \supset K_{1}, \quad D \tau_{n} K_{2} \subset K_{2}$.

There exist constants $\mu_{1}, \mu_{2}, a_{1}, a_{2}$ such that $\mu_{1 n}<\mu_{1}, \mu_{2 n}<\mu_{2}, a_{1 n}<a_{1}, a_{2 n}<a_{2}$ and the following inequalities hold:

(C) $\mu_{1}<1$;

(C) $\sqrt{\mu_{1} \cdot \mu_{2}}+\sqrt{a_{1} \cdot a_{2}}<1$.

Then any itinerary $\Pi_{I} \stackrel{\leftarrow}{\leftarrow} \Pi_{i_{1}} \leftarrow \Pi_{i_{2}}^{\tau} \leftarrow^{\tau} \cdots$ determines the smooth curve

$$
\Lambda_{i_{1} i_{2} \cdots i_{n} \cdots}^{\prime}=\bigcap_{k=1}^{\infty} \tau^{k} \Pi_{i_{k}}
$$

and $\Lambda^{\prime}$ coincides with the union of such curves. Besides any tangent vector $\mathbf{v}=(\xi, \eta)$ to $\Lambda_{i_{1} i_{2} \cdots i_{n}}^{\prime} \ldots$ belongs to $\tau_{i_{1}} K$.

If we set $K_{1}=\{\mathbb{R}, 0\} . K_{2}=K$ then $\left(C_{1}\right)$ will hold for $K_{1}$ because of the invariance of the horizontal foliation and for $K_{2}$ because of (11) and (12). Now (8), (9), (10) imply that $\left(\mathrm{C}_{2}\right),\left(\mathrm{C}_{3}\right)$ hold with

$$
\mu_{1}=\lambda^{2}, \quad \mu_{2}=\left(c_{3} A\right)^{-1}, \quad a_{1}=0, \quad a_{2}=\lambda\left(c_{3} A\right)^{-1} .
$$

Thus Alexeyev's theorem is applicable. The scheme $\left\{\Pi_{n}, \tau \Pi_{n}\right\}$ is not separable in the sense of $[1]$ because

$$
\tau\left(\Pi_{n+}\right) \cap \tau\left(\Pi_{n-}\right) \neq \varnothing \quad \text { for large } n .
$$

However, the images $\tau_{n+} K$ and $\tau_{n-} K$ do not intersect for all $n$. It follows from the subsequent inequalities where we use the notations of (11) and (12).

For $(x, y) \in \Pi_{n-}$ we have

$$
\xi_{1} / \eta_{1}=\left[\lambda^{n-1} /\left(g^{n}(y)\right)^{\prime}\right](1+\lambda(\xi / \eta))<-\frac{\lambda^{n-1}}{c_{4} \cdot 2^{n}}(1-\lambda)
$$

while for $(x, y) \in \Pi_{n+}$ we have

$$
\xi_{1} / \eta_{1}>\frac{\lambda^{n-1}}{c_{4} \cdot 2^{n}}(1-\lambda)
$$


5. Let us denote by $\pi^{\prime}: \tilde{I} \rightarrow \Lambda^{\prime}$ the map analogous to $\pi$, namely for $\tilde{x}=\left(x_{0}, x_{1}, x_{2}, \cdots\right)$ where $x_{n} \in G^{-1} x_{n-1}$,

$$
\pi^{\prime}(x)=\xi_{x_{0}} \cap \tau \xi_{x_{1}} \cap \tau^{2} \xi_{x_{2}} \cap \cdots .
$$

The map $\pi^{\prime}$ induces a homomorphism of dynamical systems

$$
\pi_{*}^{\prime}:(\tilde{I}, \tilde{G}, \tilde{\nu}) \rightarrow\left(\Lambda^{\prime}, \tau, \pi_{*}^{\prime} \tilde{\nu}\right)
$$

LeMMa 1. $\pi_{*}^{\prime}$ is an isomorphism.

Proof. For a given $J=\left(j_{1}, j_{2}, \ldots, j_{n} \ldots\right)$ consider the element $\eta_{J} \in \eta$ of the partition defined in $\S 3$. It follows from the definitions of $\eta_{J}$ and $\Lambda_{J}^{\prime}$ that $\pi^{\prime}\left(\eta_{J}\right)=\Lambda_{K}^{\prime}$ and for $\tilde{z}=\left(z_{0}, z_{1}, \ldots\right) \in \eta_{J}, \pi^{\prime}(\tilde{z})$ coincides with the projection of $z_{0} \in I$ to the curve $\Lambda_{J}^{\prime}$ along the $x$ axis. In consequence of (6) and of estimates (11) and (12) we obtain that the induced measures $\pi_{*}^{\prime} \tilde{\nu}\left(\cdot \mid \eta_{J}\right)$ are absolutely continuous with respect to the Lebesgue measure (normalized length) on $\Lambda_{J}^{\prime}$.

Let

and let

$$
\sigma_{J}=\left\{\tilde{z}: \operatorname{card} \pi^{\prime-1}\left(\pi^{\prime} \tilde{z}\right)>1\right\}
$$

$$
\pi^{\prime}\left(\sigma_{J}\right)=S_{J} \subset \Lambda_{J}^{\prime} .
$$

We show that mes $S_{J}=0$ (mes is the Lebesgue measure $\Lambda_{J}^{\prime}$ ). We have

$$
S_{J}=\bigcup_{I \neq J} \Lambda_{I}^{\prime} \cap \Lambda_{J}^{\prime} \subset \bigcup_{k=1}^{\infty} \bigcup_{i_{k} \neq j_{k}}\left(\Lambda_{i_{k} i_{k+1}}^{\prime} \ldots \cap \Lambda_{j_{k} j_{k+1} \cdots}^{\prime} \ldots\right)
$$

Let us denote $\tau\left(\Lambda^{\prime} \cap \Pi_{n \pm}\right)$ by $\Lambda_{n \pm}^{\prime}$. As $\tau$ is non-singular with respect to Lebesgue measure on $\Lambda_{K}^{\prime}$ we obtain taking into account that $\Lambda_{m}^{\prime} \cap \Lambda_{k}^{\prime} \neq \varnothing$ only for $m=n_{ \pm}$, $k=n_{ \pm}$, that it suffices to check the equality

$$
\operatorname{mes}\left(\Lambda_{n_{+} j_{2} j_{3} \cdots \cap}^{\prime} \cap \Lambda_{n_{-}}^{\prime}\right)=0 .
$$

For $\lambda<\frac{1}{2}$ the intersection $\Lambda \cap \xi_{y}$ is a Cantor set of zero Lebesgue measure for any $\xi_{y}$. Now it follows from (13) and (14) that the angles between the curves $\Lambda_{n+i_{2} i_{3}}^{\prime} \cdots$ and $\Lambda_{n-i_{2} i_{3} \ldots}^{\prime}$ are uniformly bounded away from zero. This implies (16). Thus $\operatorname{mes} S_{J}=0$. Thereby we have

$$
\tilde{\nu}\left\{y\left(\tilde{z}_{J} \mid \eta_{J}\right)=0 \text { for any } \eta_{J}\right.
$$

and the Fubini theorem gives

$$
\tilde{\nu}\left\{\tilde{z} \in \tilde{I} \mid \operatorname{card} \pi^{\prime-1}\left(\pi^{\prime} \tilde{z}\right)>1\right\}=0,
$$

and thus $\pi_{*}^{\prime}$ is an isomorphism.

Let $\tilde{\Delta}_{n \pm}=\left\{\tilde{y}=\left(y_{0}, y_{1}, \ldots\right) \in \tilde{Y} \mid y_{0} \in \Delta_{n \pm}\right\}$. If we neglect a set of zero $\tilde{\mu}$-measure we can represent $\tilde{Y}$ in the following form

$$
\tilde{Y}=\bigcup_{n} \bigcup_{0 \leq k \leq n-1} \tilde{g} \tilde{\Delta}_{n \pm}
$$

As $\pi^{\prime} \tilde{I}$ coincides with $\pi\left\{\tilde{y}=\left(y_{0}, y_{1}, \ldots\right) \mid y_{0} \in I\right\}$ we have

$$
\Lambda=\bigcup_{n} \bigcup_{0 \leq k \leq n-1} F^{k}\left(\Lambda^{\prime} \cap \Pi_{n \pm}\right)
$$


Since $F^{k}$ are non-singular with respect to Lebesgue measure on $\Lambda_{J}^{\prime}$ we obtain an isomorphism $(\tilde{Y}, \tilde{g}, \tilde{\mu}) \approx\left(\Lambda, F, \pi_{*} \tilde{\mu}\right)$ where $\pi_{*} \tilde{\mu}$ has absolutely continuous conditional measures on the curves $F^{i} \Lambda_{J}^{\prime}$ constituting the attractor $\Lambda$. According to the results of Ledrappier $[10]$ the automorphism $(\tilde{Y}, \tilde{g}, \tilde{\mu})$ is Bernoulli. Thus $\left(\Lambda, F, \pi_{*} \tilde{\mu}\right)$ is also Bernoulli and theorem 1 is proved.

Remark Although (18) defines $\Lambda$ as a union of $\Lambda_{J}^{\prime}$ and their images $F^{i} \Delta_{J}^{\prime}$, it is easy to verify that every leaf of $\Lambda$ is a smooth curve which connects the top of the square $S$ to its bottom. These curves may be identified as

$$
\Lambda_{p_{1} p_{2} p_{3} \ldots}=\pi\left\{\tilde{y}=\left(y_{0}, y_{1}, y_{2}, \ldots\right) \mid y_{0} \in[0,1], y_{1}=g_{p_{1}}^{-1}\left(y_{0}\right), y_{2}=g_{p_{2}}^{-1}\left(y_{1}\right), \ldots\right\}
$$

where $p_{i} \in\{0,1\}, g_{0}^{-1}(x) \in\left[0, \frac{1}{2}\right], g_{1}^{-1}(x) \in\left[\frac{1}{2}, 1\right]$.

6. Now we turn to the proof of theorem 2 . We consider again a map of the square given by (1)

$$
F:(x, y) \rightarrow\left(\varphi(y)+\lambda\left(x-\frac{1}{2}\right), g(y)\right)
$$

where $\varphi(y)$ is the same as in $\S 2$ and $g \in C^{3}([0,1],[0,1])$ which satisfies (i) but instead of (ii) we assume $\max g(y)=g(c)<1$.

Invariant measures for such mappings were studied in [8]. It follows from [8] that for the family $y \rightarrow a y(1-y)$ and for any family $y \rightarrow g_{a}(y)$ sufficiently close to $y \rightarrow a y(1-y)$ in $C^{3}([0,4] \times[0,1],[0,1])$ there is a set of parameter values $\mathfrak{M}$ such that mes $\mathfrak{M}>0$ and for $a \in \mathfrak{M}$ the map $g_{a}$ has an absolutely continuous invariant measure. In contrast with the situation considered in $\$ \$ 2-5$ we cannot prove the existence of an absolutely continuous Bernoulli measure for any $\lambda \in\left[0, \lambda_{0}\right]$ although we think it is true. Instead we will prove it for $\lambda$ belonging to some subset of full measure. Let a family $\left\{g_{a}\right\}$ and a set $\mathfrak{M}$ be as above.

THEOREM 2. For the family

$$
F_{a}:(x, y) \rightarrow\left(\varphi(y)+\lambda\left(x-\frac{1}{2}\right), g_{a}(y)\right)
$$

there is a set $\Xi \subset\left[0, \lambda_{0}\right]$ such that mes $\Xi=\lambda_{0}$ and for any $\lambda \in \Xi$ the subset $\mathfrak{M}^{\prime}$ of $\mathfrak{M}$ defined by

$$
\mathfrak{M}^{\prime}=\left\{a: \text { the attractor of } F_{a} \text { admits an a.c.B. measure }\right\}
$$

satisfies mes $\left(\mathfrak{M} \backslash \mathfrak{M}^{\prime}\right)=0$.

We begin with constructing the induced map $G: I \rightarrow I$ and the corresponding two-dimensional map $\tau: \Pi_{I} \rightarrow \Pi_{I}$ induced by $F$ on the strip $\Pi_{I}$. This is done exactly as in $\$ \S 3$ and 4 but now $G$ has only a finite number of monotone branches $G_{k}: D_{k} \rightarrow I$ and one central parabolic branch. Correspondingly the image $\tau \Pi_{I}$ consists of a finite number of nearly vertical strips $\tau \Pi_{D_{k}}$ and of a narrow horseshoe (figure 5). Certainly it suffices to construct the desired attractor for $\tau$.

An absolutely continuous invariant measure $\mu(d y)$ for $G$ was constructed in [8] with the help of an auxiliary map $T: I \rightarrow I$ satisfying the following conditions. There 


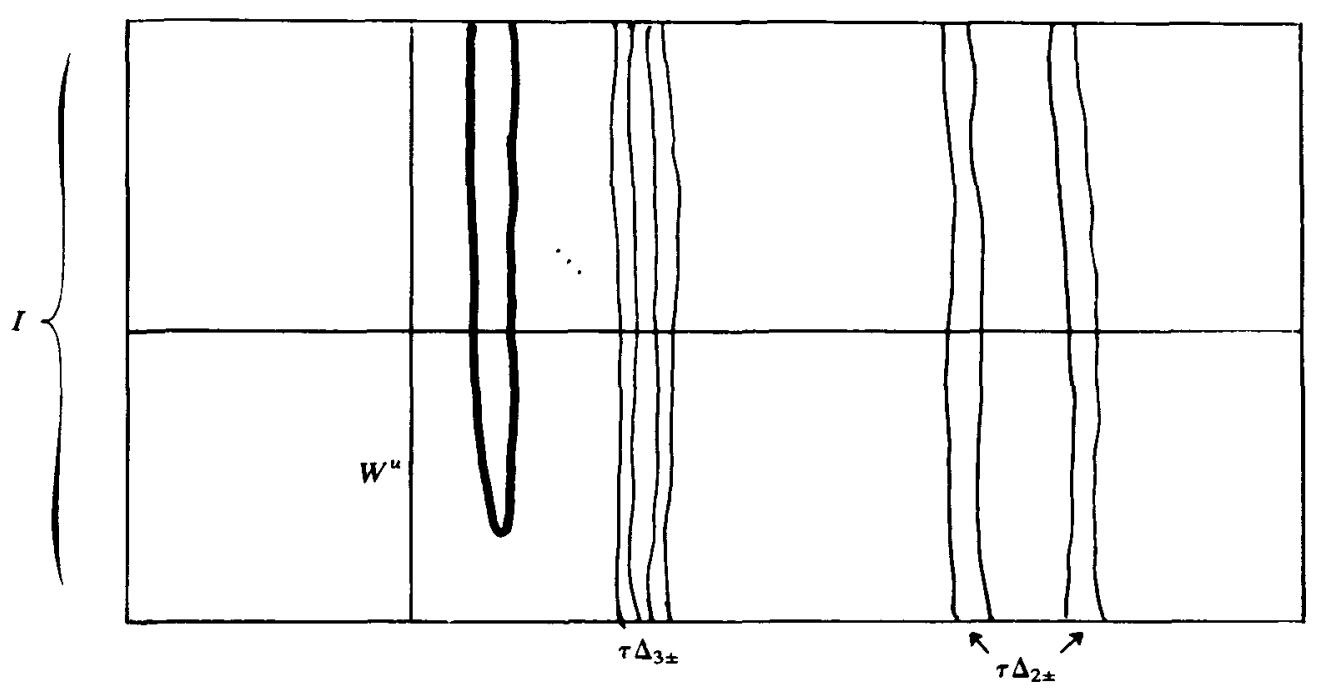

FIGURE 5

is a countable number of intervals $\Delta_{i} \subset I$ such that:

1) int $\Delta_{i} \cap$ int $\Delta_{j}=\varnothing$;

2) the Lebesgue measure of $\bigcup \Delta_{i}$ equals mes $I$;

3) $T \mid \Delta_{i}=T_{i} \approx G^{n_{i}}$ are diffeomorphisms, $\operatorname{Im} T_{i}=I$;

4) $\sup _{i} \max _{x \in \Delta_{i}}\left|D^{2} T(x) / D T(x)\right|\left(\operatorname{mes} \Delta_{i}\right)<c_{0}$;

5) $|D T(x)|>L \gg 1$;

6) $\sum_{i} n_{i}$ mes $\Delta_{i}<\infty$.

According to $[13]$ there exists a $T$-invariant Bernoulli measure $\nu(d y)$ with the continuous density $\chi(y)>0$ with respect to $d y . \nu$ generates the $G$-invariant measure $\mu$ by formula (5).

For $Z_{0}=I \backslash \cup \Delta_{i}$ set

$$
Z=\bigcup_{n \geq 0} G^{-n}\left(\bigcup_{k \geq 0} G^{k} Z_{0}\right)
$$

Then $\mu(Z)=0, G^{-1}(Z)=G(Z)=Z$. Consider $X=I \backslash Z$. Then the endomorphisms $(I, G, \mu)$ and $(X, G, \mu)$ are isomorphic and for any $x \in X, k \in \mathbb{Z}$ and $x^{\prime} \in G^{k}(x) T(x)$ is defined. We shall use the notation $(\tilde{I}, \tilde{T}, \tilde{\mu})$ for the natural extension of $T$, and $(\tilde{X}, \tilde{G}, \tilde{\mu})$ for the natural extension of $G$. Let $\mathscr{C}$ be the corresponding twodimensional map which coincides with $\mathscr{C}_{i}=\tau^{n_{i}}$ on $\Pi_{\Delta_{i}}$.

Let us denote by $\Delta=\bigcap_{n=0}^{\infty} \tau^{n} \Pi_{I}$ the attractor for the map $\tau$; by $\Lambda^{\prime}=\bigcap_{n=0}^{\infty} \mathscr{C}^{n} \Pi_{I}$ the attractor for $\mathscr{C}$. We define the map $\pi: \tilde{X} \rightarrow \Lambda$ by

$$
\pi\left(\tilde{x}=\left(x_{0}, x_{1}, x_{2}, \ldots\right)\right)=\xi_{x_{0}} \cap \tau \xi_{x_{1}} \cap \tau^{2} \xi_{x_{2}} \cap \cdots
$$

and the map $\pi^{\prime}: \tilde{I} \rightarrow \Lambda^{\prime}$ by

$$
\pi^{\prime}\left(\tilde{y}=\left(y_{0}, y_{1}, y_{2}, \ldots\right)\right)=\xi_{y_{0}} \cap \mathscr{C} \xi_{y_{1}} \cap \mathscr{C}^{2} \xi_{y_{2}} \cap \cdots .
$$

7. Let $\mathscr{A}$ be the partition of $\tilde{I}$ into cylinders $\left(\Delta_{i}, T^{-1} \Delta_{i}, T^{-2} \Delta_{i}, \ldots\right)$. Consider the corresponding decreasing partition $\bigvee_{n=1}^{\infty} \tilde{T}^{n}=\eta$ and the increasing one 
$\bigvee_{n=0}^{\infty} T^{-n} \mathscr{A}=\gamma$. Let us denote by $\alpha$ the measure induced by $\tilde{\nu}$ on the factor space $\tilde{I} / \eta$. Let $C_{\eta}$ be an element of $\eta ; \nu\left(\cdot \mid C_{\eta}\right)$ the conditional measure induced by $\tilde{\nu}$ on $C_{\eta} ; C_{\gamma}$ an element of $\gamma ; \nu\left(\cdot \mid C_{\gamma}\right)$ the conditional measure on $C_{\gamma}$. The following assertion may be considered as a simple version of several theorems about systems with absolutely continuous foliations (see e.g. $[3,11]$ ).

LEMMA 2. $\tilde{\nu}$ is equivalent to the direct product $\nu \times \alpha$. There are constants $c_{1}, c_{2}>0$ so that for any $\nu$-measurable set $\Delta \subset I$ and for any $\alpha$-measurable set $\Gamma \subset \tilde{I} / \eta$ one has

$$
c_{2}<\frac{\tilde{\nu}(\Delta \times \Gamma)}{\nu(\Delta) \cdot \alpha(\Gamma)}<c_{1} .
$$

Proof. As $\tilde{T}^{-n} \mathscr{A}=\left\{\left(T^{-n} \Delta_{i}, T^{-(n+1)} \Delta_{i}, \ldots\right)\right.$ we have

$$
\bigvee_{0}^{N} \tilde{T}^{-n} \mathscr{A}=\left\{\Delta_{i_{0} i_{1} \cdots i_{N}}, T^{-1} \Delta_{i_{0} i_{1} \cdots i_{N}}, \cdots\right\}
$$

where

$$
\Delta_{i_{0} i_{1} \cdots i_{N}}=\Delta_{i_{0}} \cap T^{-1} \Delta_{i_{1}} \cap \cdots \cap T^{-N} \Delta_{i_{N}}
$$

The intersection $\Delta_{i_{0}} \cap T^{-1} \Delta_{i_{1}} \cap \cdots \cap T^{-N} \Delta_{i_{N}} \cap \cdots$ is a point for any sequence $\left(i_{0}, i_{1}, i_{2}, \ldots i_{N} \ldots\right)$ because of 5$)$. Thus we obtain

$$
\gamma=\bigvee_{0}^{\infty} \tilde{T}^{-n} \mathscr{A}=\left\{\left(y, T^{-1} y, T^{-2} y, \ldots\right)\right\}
$$

On the other hand

Hence

$$
\tilde{T}^{n} \mathscr{A}=\left\{(\underbrace{I, I, \ldots, I}_{n \text { times }}, \Delta_{i}, T^{-1} \Delta_{i}, \ldots)\right\}
$$

$$
\bigvee_{1}^{N} \tilde{T}^{n} \mathscr{A}=\left\{\left(I, \Delta_{i_{1}}, \Delta_{i_{2} i_{1}}, \ldots, \Delta_{i_{N i_{N-1}} \cdots i_{1}}, T^{-1} \Delta_{i_{N i_{N-1}} \cdots i_{1}}, \ldots\right)\right\}
$$

and we obtain

$$
\eta=\bigvee_{1}^{\infty} \tilde{T}^{n} \mathscr{A}=\left\{\left(I, \Delta_{i_{1}}, \Delta_{i_{2} i_{1}}, \ldots, \Delta_{i_{N i_{n-1} \cdots i_{1}}}, \Delta_{i_{N+1} i_{N} \cdots i_{1}}, \ldots\right) .\right.
$$

Any point $\tilde{y}=\left(y_{0}, y_{1}, y_{2}, \ldots, y_{n}, \ldots\right) \in \tilde{I}$ may be uniquely represented as $\tilde{y}=$ $C_{\eta} \subset C_{\gamma}$ where the indices $i_{1}, i_{2}, \ldots$ in the definition of $C_{\eta}$ are determined by $y_{n} \in \Delta_{i_{n} \cdots i_{1}}$ and $C_{y}=\left(y_{0}, T^{-1} y_{0}, \ldots\right)$. This gives the direct product structure in $\tilde{I}$. Let $\Delta \subset I$ be a measurable set, $\Gamma_{\Delta}=\left(\Delta, T^{-1} \Delta, T^{-2} \Delta, \ldots\right), \Gamma_{i_{1} i_{2} \cdots i_{n}}$ the cylinder in $\tilde{I}$ defined by

$$
\Gamma_{i_{1} i_{2} \cdots i_{n}}=\left(I, \Delta_{i_{1}}, \Delta_{i_{2} i_{1}}, \ldots, \Delta_{i_{n} \cdots i_{2} i_{1}}, T^{-1} \Delta_{i_{n} \cdots i_{2} i_{1}}, T^{-2} \Delta_{i_{n} \cdots i_{2} i_{1}}, \cdots\right)
$$

Let us denote the intersection $\Gamma_{\Delta} \cap \Gamma_{i_{1} i_{2} \cdots i_{n}}$ by $P\left(\Delta,\left[i_{1}, \ldots, i_{n}\right]\right)$. We shall use the notation $T_{i_{1} i_{2} \cdots i_{k}}$ for the unique monotone branch of $T^{k}$ which maps

$$
\Delta_{i_{k}} \cap T^{-1} \Delta_{i_{k-1}} \cap \cdots \cap T^{-(k-1)} \Delta_{i_{1}}
$$

onto $I$. The assertion of lemma 2 will be proved if we show that independently from $\Delta$ and $\left[i_{1} i_{2} \cdots i_{n}\right]$ the following inequality holds:

$$
c_{2}<\frac{\tilde{\nu}\left(P\left(\Delta,\left[i_{1} i_{2} \cdots i_{n}\right]\right)\right)}{\tilde{\nu}\left(\Gamma_{\Delta}\right) \cdot \tilde{\nu}\left(\Gamma_{i_{1} i_{2} \cdots i_{n}}\right)}<c_{1} .
$$


According to the definition of $\tilde{\nu}$ we have

$$
\begin{aligned}
\tilde{\nu}\left(\Gamma_{\Delta}\right) & =\nu(\Delta), \\
\tilde{\nu}\left(\Gamma_{i_{1} i_{2} \cdots i_{n}}\right) & =\nu\left(T_{i_{1} i_{2} \cdots i_{n}}^{-n}(I)\right), \\
\tilde{\nu}\left(P\left(\Delta,\left[i_{1} i_{2} \cdots i_{n}\right]\right)\right) & =\nu\left(T_{i_{1} i_{2} \cdots i_{n}}^{-n}(\Delta)\right) .
\end{aligned}
$$

Thus (20) is equivalent to

$$
c_{2}<\frac{\nu\left(T_{i_{1} i_{2} \cdots i_{n}}^{-n}(\Delta)\right)}{\nu\left(T_{i_{1} i_{2} \cdots i_{n}}^{-n}(I)\right)}: \frac{\nu(\Delta)}{\nu(I)}<c_{1} .
$$

Now it follows from properties 4) and 5) of $T$ that 4) holds not only for $T_{i}$ but also for all their compositions $T_{i_{1}} \circ T_{i_{2}} \circ \cdots \circ T_{i_{n}}$ with a constant $c_{5}$ independent from $\left[i_{1} i_{2} \cdots i_{n}\right]$, see for example lemma 5 of $[8]$. Thus $b_{1}<D T^{n}(x) / D T^{n}(y)<b_{2}$ for some $b_{1}, b_{2}>0$; and for any $x, y \in \Delta_{i_{n} \cdots i_{1}}$. This implies an inequality similar to (21) but for Lebesgue measure. Since $0<d_{1}=\min _{y \in I} \chi(y)<\nu(A) /$ mes $A<$ $\max _{y \in I} \chi(y)=d_{2}$ for any measurable set $A$ we obtain (21) which finishes the proof of lemma 2 .

COROLlaRY. Let $\theta_{1}: \tilde{I} \rightarrow I$ be the projection along $C_{\gamma}$ and $\theta_{2}: \tilde{I} \rightarrow \tilde{I} / \eta$ the projection along $C_{\eta}$. Then for any element $C_{\eta}$ and for any measurable $\Delta \subset C_{\eta}$

$$
c_{2}<\frac{\tilde{\nu}\left(\Delta \mid C_{\eta}\right)}{\nu\left(\theta_{1} \Delta\right)}<c_{1} .
$$

Similarly for any measurable $\Gamma \subset \tilde{I} / \eta$

$$
c_{2}<\frac{\tilde{\nu}\left(\Gamma \mid C_{\gamma}\right)}{\alpha\left(\theta_{2} \Gamma\right)}<c_{1} .
$$

In particular we obtain that $\eta$ and $\gamma$ are absolutely continuous partitions and the intersection $\eta \wedge \gamma$ is trivial.

8. The following construction which clarifies the relation between $(\tilde{X}, \tilde{G}, \tilde{\mu})$ and $(\tilde{I}, \tilde{T}, \tilde{\nu})$ is similar to the 'tower' construction for automorphisms. For any $\Delta_{i}$ consider the sets

$$
A_{i k}=G^{k} \Delta_{i}, \quad 0 \leq k \leq n_{i},
$$

and let $\mathscr{X}=\bigcup_{i, k} A_{i k}$ be the disjoint sum of $A_{i k}$. We define the projection $p: \mathscr{X} \rightarrow I$ by assigning to any $u \in A_{i k}$ its image under the natural inclusion $p(u) \in G^{k} \Delta_{i} \subset I$. Then we define a map $\mathscr{F}: \mathscr{X} \rightarrow \mathscr{X}$. If $u \in A_{i k}, k<n_{i}-1$, then

if $u \in \boldsymbol{A}_{i n_{i}-1}$ then

$$
\mathscr{F}(u)=p^{-1}(G(u)) \cap A_{i k+i} \text {; }
$$

$$
\mathscr{F}(u)=p^{-1}(G(u)) \cap \bigcup_{j} A_{j 0}
$$

The construction implies $G \circ p=p \circ \mathscr{F}$. Notice that if we identify $\bigcup_{i} A_{i 0}$ with $I$, then the map induced by $\mathscr{F}$ on $I \subset \mathscr{X}$ coincides with $T$.

Let us define the measure $\rho$ on $\mathscr{Z}$ by

$$
\rho\left(U \subset A_{i k}\right)=\nu\left(\mathscr{F}^{-k} U\right) .
$$


Since $\nu$ is $T$-invariant $\rho$ is $\mathscr{F}$-invariant, and the definition of $\mu$ implies that $p_{*} \rho=\mu$. Consider the natural extension $(\tilde{\mathscr{X}}, \tilde{\mathscr{F}}, \tilde{\rho})$. It follows from

$$
G \circ p=p \circ \mathscr{F}
$$

that the map $\tilde{p}: \tilde{\mathscr{X}} \rightarrow \tilde{X}$ defined by $\tilde{p}\left(u_{0}, u_{1}, \ldots\right)=\left(p u_{0}, p u_{i}, \ldots\right)$ satisfies

$$
\tilde{p} \circ \tilde{F}=\tilde{G} \circ \tilde{p} .
$$

LeMma 3. $\tilde{P}$ is an isomorphism of dynamical systems $(\tilde{\mathscr{X}}, \tilde{\mathscr{F}}, \tilde{\rho})$ and $(\tilde{X}, \tilde{G}, \tilde{\mu})$.

Proof. We show that there is a subset $\tilde{M}$ of $\tilde{X}$ such that $\tilde{\mu}(\tilde{M})=0$ and $\tilde{p}: \tilde{\mathscr{X}} \rightarrow \tilde{X} \backslash \tilde{M}$ is one-to-one. For $x \in \Delta_{k}$ set $n(x)=n_{k}$. We define $\tilde{M}=\bigcup_{i=0}^{\infty} \tilde{M}_{i}$ where the set $\tilde{M}_{i}$ is defined by

$$
\tilde{x}=\left(x_{0}, x_{1}, \ldots, x_{i}, x_{i+1}, \ldots\right) \in \tilde{M}_{i}
$$

iff there is a sequence $j_{n} \rightarrow \infty$ so that $n\left(x_{j_{n}}\right)>j_{n}-i$. Since $n\left(x_{j_{n}}\right)>j_{n}-i \rightarrow \infty$ we obtain for $\tilde{x} \in \tilde{M}_{i}$ that $x_{j_{n}} \in \Delta_{p_{n}}$ where $p_{n} \rightarrow \infty$ when $n \rightarrow \infty$. Now.

$$
\tilde{\nu}\left\{\tilde{x}: x_{j_{n}} \in \Delta_{p_{n}}\right\}=\mu\left(\Delta_{p_{n}}\right)
$$

hence it is sufficient to show that

$$
\lim _{N \rightarrow \infty}\{x: n(x)>N\}=0 .
$$

But this follows from the convergence of $\sum_{k=1}^{\infty} \mu\left(\Delta_{k}\right)$. Thus $\mu\left(\tilde{M}_{i}\right)=0$ and consequently $\tilde{\mu}(\tilde{M})=0$.

We shall use the following decomposition property which follows from the inductive construction of the maps $T_{i}$ in [8].

Property D. Let $T \mid \Delta_{i}=T_{i}=G^{n_{i}}$. Then for every $k<n_{i}$ there are $T_{i_{1}}, \ldots, T_{i r}, r \geq 1$, so that the restriction of $G^{n_{i}-k}$ to $G^{k} \Delta_{i}$ may be represented as

$$
G^{n_{i}-k}=T_{i_{1}} \circ T_{j_{2}} \circ \cdots \circ T_{j,} \text {. }
$$

Consider a point $\tilde{x}=\left(x_{0}, x_{1}, \ldots\right) \in \tilde{X} \backslash \tilde{M}$. From $\tilde{x} \notin \tilde{M}_{0}$ we obtain that there exists

$$
m_{0}(\tilde{x})=\max \left\{m: n\left(x_{m}\right)>m\right\} .
$$

Then property D implies $n\left(x_{m_{0}+k}\right) \leq k, k=1,2, \ldots$ In particular, $n\left(x_{m_{0}+1}\right)=1$.

For $k \geq 1$ we define

$$
m_{k}=\max _{i>m_{k-1}}\left\{i: n\left(x_{i}\right)=i-m_{k-i}\right\}
$$

Property D implies

$$
i-n\left(x_{i}\right) \geq m_{k-1} \quad \text { for } m_{k-1}<i \leq m_{k} .
$$

Let $\tilde{x}=\left(x_{0}, x_{1}, \ldots, x_{n_{0}}, \ldots, x_{n_{1}}, \ldots, x_{n_{2}}, \ldots\right)$ where $\left(n_{0}, n_{1}, n_{2}, \ldots\right)$ is the sequence defined above, $x_{n_{i}} \in \Delta_{m_{i}}$. Then $\tilde{u}=\left(u_{0}, u_{1}, \ldots, u_{n_{0}}, \ldots, u_{n_{1}}, \ldots, u_{n_{2}}, \ldots\right) \in \mathscr{Z}$ defined by

$$
u_{n_{i}}=x_{n_{i}} \in A_{i_{i} 0}, \quad u_{n_{i}-k}=\mathscr{F}^{k} u_{n_{i}}, \quad k=1, \ldots, n_{i}-n_{i-1}
$$

satisfies $\tilde{p} \tilde{u}=\tilde{x}$, hence $\tilde{p}$ is onto $\tilde{X} \backslash \tilde{M}$. Let $\tilde{p} \tilde{u}^{\prime}=\tilde{x}$, where $\tilde{u}^{\prime}=\left(j_{0}^{\prime}, j_{1}^{\prime}, \ldots\right)$. Let $n_{0}^{\prime}=\min \left\{n: u_{n}^{\prime} \in I\right\}$ and for $k \geq 1$

$$
n_{k}^{\prime}=\min \left\{n: n>n_{k-1}, u_{n}^{\prime} \in I\right\}
$$


The definition of the sequence $\left(n_{0}, n_{1}, \ldots\right)$ implies $T x_{n_{i}}=x_{n_{i}}$ and the construction of $(\mathscr{X}, \mathscr{F})$ implies $T x_{n_{i}^{\prime}}=x_{n_{i-1}}^{\prime}$. Besides it follows from the definitions of $n_{0}$ and of $n_{0}^{\prime}$ that $n\left(x_{n_{0}}\right)>n_{0}$ and $n\left(x_{n_{0}}\right)>n_{0}^{\prime}$. Suppose $n_{0}^{\prime}<n_{0}$. Then $n_{0} \in\left[n_{k}^{\prime}, n_{k+1}^{\prime}\right]$ and using property $\mathrm{D}$ we obtain $n\left(x_{n_{0}}\right) \leq n_{0}-n_{k}^{\prime}<n_{0}$, a contradiction. The same arguments show that $n_{0}<n_{0}^{\prime}$ contradicts to $n\left(x_{n_{0}}^{\prime}\right)>n_{0}^{\prime}$. Therefore, $n_{0}=n_{0}^{\prime}$. Analogously using property $\mathrm{D}$ one obtains $n_{k}=n_{k}^{\prime}$ for all $k$. Since

$$
\mathscr{F}: A_{i k} \rightarrow A_{i+i k} \text { and } G: G^{k} \Delta_{i} \rightarrow G^{k+1} \Delta_{i}
$$

are homeomorphisms for $0 \leq k \leq n_{i}-1$ we obtain $\tilde{u}^{\prime}=\tilde{u}$, and lemma 3 is proved.

Let us denote

$$
\tilde{B}=\left\{\tilde{u}=\left(u_{0}, u_{1}, \ldots\right) \in \tilde{\mathscr{X}} \mid u_{0} \in \bigcup_{i} A_{i 0}\right\} .
$$

It follows from the proof of lemma 3 that $\tilde{p} B$ coincides with $\left\{\tilde{x}=\left(x_{0}, x_{1}, \ldots\right): n_{0}(\tilde{x})=\right.$ $0\}$. As the automorphism induced by $\mathscr{F}$ on $(\tilde{B}, \tilde{\rho})$ coincides with $(\tilde{I}, \tilde{T}, \tilde{\nu})$ we obtain that $\tilde{p}$ is an isomorphism between $(\tilde{I}, \tilde{T}, \tilde{\nu})$ and the automorphism induced by $\tilde{\mathrm{G}}$ on $(\tilde{p} \tilde{B}, \tilde{\mu})$. For

$$
\tilde{y}=\left(y_{0}, y_{1}, y_{2}, \ldots\right) \in \tilde{I}, \quad \text { where } y_{i}=G^{n_{i+1}} y_{i+1},
$$

we have

$$
\tilde{p} \tilde{y}=\left(y_{0}, G^{n_{1}-1} y_{1}, \ldots, G y_{1}, y_{1}=G^{n_{2}} y_{2}, G^{n_{2}-1} y_{2}, \ldots, y_{2}=G^{n_{3}} y_{3}, \ldots\right) .
$$

Remark. The representation of $(\tilde{I}, \tilde{T}, \tilde{\nu})$ as an induced automorphism, the triviality of the intersection $\eta \wedge \gamma$ proved in $\S 7$, and the $K$-property of $T$ allow us to apply the results of Gurevich [6] and Blanchard [5] which give the following corollary.

COROllary. $(\tilde{X}, \tilde{G}, \tilde{\mu})$ is a $K$-system.

This follows of course from the B-property proved by Ledrappier in [10]. The above construction gives another way to construct the unstable foliation.

9. Let us consider a point

$$
x=\left(x_{0}=x_{n_{0}}, x_{1}, \ldots, x_{n_{1}}, \ldots, x_{n_{2}}, \ldots\right) \in \tilde{X}
$$

and its pre-image

$$
\tilde{p}^{-1} \tilde{x}=\left(x_{n_{0}}, x_{n_{1}}, x_{n_{2}}, \ldots\right) \in \tilde{I} .
$$

For the projections $\pi$ and $\pi^{\prime}$ defined in $\S 6$ we have

$$
\begin{aligned}
\pi(\tilde{x}) & =\xi_{x_{0}} \cap \tau \xi_{x_{1}} \cap \cdots \cap \tau^{n_{1}} \xi_{x_{n_{1}}} \cap \cdots \cap \tau^{n_{1}+n_{2}} \xi_{x_{n_{2}}} \cap \cdots \\
& =\xi_{x_{n_{0}}} \cap \tau^{n_{1}} \xi_{x_{n_{1}}} \cap \tau^{n_{1}+n_{2}} \xi_{x_{n_{2}}} \cap \cdots \\
& =\pi^{\prime}\left(\tilde{p}^{-1} \tilde{x}\right) .
\end{aligned}
$$

Thus the image of $\tilde{I}$ under $\pi^{\prime}$ coincides with its image under $\pi$ when we consider $\tilde{I}$ as the subset of $\tilde{X}$. Using the result of the previous section we represent $\left(\Lambda^{\prime}, \mathscr{C}, \pi_{*}^{\prime} \tilde{\nu}\right)$ as an induced system with respect to $\left(\Lambda, \tau, \pi_{*} \mu\right)$. 
Now we check that $\left\{\Pi_{\Delta_{i}}, \mathscr{C}_{i}\right\}$ satisfy the conditions of Alexeyev's theorem. For $T: \Delta \rightarrow I$ and the corresponding $\mathscr{C}: \Pi_{\Delta} \rightarrow \Pi_{I}$ where $T=G^{r}, \mathscr{C}=\tau^{r}$, we can rewrite $(8),(9)$ as

$$
D \tau(x, y)=\left(\begin{array}{cc}
\lambda^{m} & \lambda^{m-1} \varphi^{\prime}(y) \\
0 & G^{\prime}(y)
\end{array}\right)
$$

where $y \in \Pi_{m \pm}, G(y)=g^{m}(y)$ and $\varphi^{\prime}(y) \equiv 1$ for $m>2$. Consider a point $\left(x_{r}, y_{r}\right) \in \Pi_{\Delta}$ and for $k \in[0, r]$ set

$$
\left(x_{k}, y_{k}\right)=\tau^{r-k}\left(x_{r}, y_{r}\right)
$$

in particular

$$
\left(x_{0}, y_{0}\right)=\mathscr{C}\left(x_{r}, y_{r}\right)=\tau^{r}\left(x_{r}, y_{r}\right)
$$

Then

$$
\begin{aligned}
& D \mathscr{C}\left(x_{r}, y_{r}\right)=\prod_{i=1}^{r}\left(\begin{array}{cc}
\lambda^{m_{i}} & \lambda^{m_{i}-1} \varphi^{\prime}\left(y_{i}\right) \\
0 & G^{\prime}\left(y_{i}\right)
\end{array}\right) \\
& =\left(\begin{array}{cc}
\lambda^{\sum_{i=1}^{\prime} m_{i}} & \lambda^{-1}\left[\lambda^{m_{1}} \varphi^{\prime}\left(y_{1}\right) \prod_{i=2}^{r} G^{\prime}\left(y_{i}\right)+\cdots+\lambda^{\sum_{i-1}^{r-1} m_{i}} \varphi^{\prime}\left(y_{r-1}\right) G^{\prime}\left(y_{r}\right)+\lambda^{\sum_{i=1}^{r} \varphi^{\prime}\left(y_{r}\right)}\right] \\
0 & \prod_{i=1}^{r} G^{\prime}\left(y_{i}\right)
\end{array}\right)
\end{aligned}
$$

Consequently

$D \mathscr{C}\left(x_{r}, y_{r}\right)(\xi, \eta)=\left(\xi_{1}, \eta_{1}\right)$

$$
\begin{aligned}
= & \left(\lambda^{\sum_{i=1}^{r} m_{i}} \xi+\lambda^{-1}\left(\lambda^{m_{1}} \varphi^{\prime}\left(y_{1}\right)\right.\right. \\
& \left.\times \prod_{i=2}^{r} G^{\prime}\left(y_{i}+\cdots+\lambda^{\sum_{1}^{r_{1} m_{i}}} \varphi^{\prime}\left(y_{r}\right)\right) \eta, \prod_{i=1}^{r} G^{\prime}\left(y_{i}\right) \eta\right) .
\end{aligned}
$$

Hence

$$
\frac{\xi_{1}}{\eta_{1}}=\lambda^{-1}\left(\frac{\lambda^{m_{1}} \varphi^{\prime}\left(y_{1}\right)}{G^{\prime}\left(y_{1}\right)}+\frac{\lambda^{m_{1}+m_{2}} \varphi^{\prime}\left(y_{2}\right)}{G^{\prime}\left(y_{1}\right) G^{\prime}\left(y_{2}\right)}+\cdots+\frac{\lambda^{\sum_{1}^{r_{1}} m_{i}} \varphi^{\prime}\left(y_{r}\right)}{\prod_{i=1}^{r} G^{\prime}\left(y_{i}\right)}\right)+\frac{\lambda^{\sum_{1}^{\prime \prime} m_{i}}}{\prod_{i=1}^{r} G^{\prime}\left(y_{i}\right)} \cdot \frac{\xi}{\eta} .
$$

According to property $\mathrm{D} G^{k} \mid G^{r-k} \Delta$ may be represented as a composition

$$
G^{k}=T_{s_{1}} \circ \ldots \circ T_{s_{1}} \text {. }
$$

Since $\left|D T_{j}\right|>L$ for any $j$ we have

$$
\left|G^{\prime}\left(y_{1}\right) \circ \cdots \circ G^{\prime}\left(y_{k}\right)\right|=\left|\left(G^{k}\right)^{\prime}\right|>L^{\prime} .
$$

Thus $\left|\xi_{1} / \eta_{1}\right|$ may be estimated as

$$
\left|\xi_{1} / \eta_{1}\right|<\lambda^{m_{1}-1} / L(1-\lambda)
$$

It follows from (23) that for any $\mathscr{C}_{i}=\mathscr{C} \mid \Pi_{\Delta_{i}}$ the cone $|\xi / \eta|<1$ is invariant under $D \mathscr{C}_{i}$. The remaining conditions of Alexeyev's theorem are also satisfied with

$$
\mu_{1}=\lambda^{2}, \quad \mu_{2}=L^{-1}, \quad a_{1}=0, \quad a_{2}=\lambda L^{-1} .
$$


Therefore $\left\{\Pi_{\Delta_{i}}, \mathscr{C}_{i}\right\}$ form an itinerary scheme. This implies just as in $\S 4$ that $\Lambda^{\prime}$ is the union of smooth curves $\Lambda_{J}^{\prime}=\Lambda_{j_{1} j_{2} \cdots j_{n}}^{\prime} \cdots$. Any $\Lambda_{J}^{\prime}$ is a graph of a function $x(y)$ satisfying $|d x / d y|<\lambda / L(1-\lambda)$. For an element $C_{J}$ of the partition $\eta$ defined by

$$
C_{J}=\left(I, \Delta_{j_{1}}, \Delta_{j_{2} j_{1}}, \ldots\right)=\left\{\left(y, T_{j_{1}}^{-1} y, T_{j_{2}}^{-1} \circ T_{j_{1}}^{-1} y, \ldots\right)\right\}, \quad y \in I,
$$

we have $\pi^{\prime}\left(C_{J}\right)=\Lambda_{J}^{\prime}$ and $\pi^{\prime}\left(y, T_{j_{1}}^{-1} y, \ldots\right)$ coincides with the projection of $y$ on $\Lambda_{J}^{\prime}$ along the $x$-axis. Since the conditional measure $\tilde{\nu}\left(\cdot \mid C_{J}\right)$ is equivalent to $d y$, the conditional measure $\pi_{*}^{\prime}\left(\cdot \mid \Lambda_{J}^{\prime}\right)$ is equivalent to Lebesgue measure on $\Lambda_{J}^{\prime}$. Set $\Lambda_{\Delta_{i}}^{\prime}=$ $\Lambda^{\prime} \cap \Pi_{\Delta_{i}}$.

The representation of $\left(\Lambda^{\prime}, \mathscr{C}\right)$ as an induced system with respect to $(\Lambda, \tau)$ gives

$$
\Lambda=\bigcup_{i} \bigcup_{k=0}^{n_{i}-1} \tau^{k}\left(\Lambda_{\Delta_{i}}^{\prime}\right) \text {. }
$$

Notice that contrary to (18) all the summands in (24) are contained in $\Pi_{I}$. One can check that the leaves or $\Lambda$ are smooth curves but their projections on the $y$-axis depend now on the curves; the turning points lie on $\xi_{c_{n}}$ where $c_{n}$ belong to the trajectory of the critical point $c$.

10. Now we take into account the dependence on $a$ and $\lambda$. For a map

$$
F_{a, \lambda}(x, y)=\left(\varphi(y)+\lambda\left(x-\frac{1}{2}\right), g_{a}(y)\right)
$$

let $\left(\tilde{X}_{a}, \tilde{G}_{a}, \tilde{\mu}_{a}\right)$ and $\left(\Lambda_{a, \lambda}, \tau_{a, \lambda}, \xi_{a \lambda *} \tilde{\mu}_{a}\right)$ be the corresponding systems. The preceding results were obtained for any $a \in \mathfrak{M}$ and any $0<\lambda \leq \lambda_{0}$. Let

$$
\sigma_{a, \lambda}=\left\{\tilde{x} \in \tilde{X}_{a}: \operatorname{card} \pi^{-1}(\pi(x))>1\right\} .
$$

LEMMA 4. (i) For any $a \in \mathfrak{M}$ and for any $\lambda \in\left(0, \lambda_{0}\right]$ either

$$
\tilde{\mu}_{a}\left(\sigma_{a \lambda}\right)=0 \text { or } \tilde{\mu}_{a}\left(\tilde{\sigma}_{a \lambda}\right)=\tilde{\mu}_{a}\left(\tilde{X}_{a}\right) \text {; }
$$

(ii) for any $a \in \mathfrak{M}$

$$
\operatorname{mes}\left\{\lambda: \tilde{\mu}_{a}\left(\sigma_{a \lambda}\right)=\tilde{\mu}_{a}\left(\tilde{X}_{a}\right)\right\}=0 .
$$

Let us show that lemma 4 implies theorem 2. Consider the measure $d a \times d \lambda$ on the direct product $\mathfrak{R} \times\left(0, \lambda_{0}\right]$. Let

$$
\Xi=\left\{\lambda \in\left(0, \lambda_{0}\right]: \operatorname{mes}\left\{a \in \mathfrak{M}: \tilde{\mu}_{a}\left(\sigma_{a \lambda}\right)>0\right\}=0\right\} .
$$

It follows from the Fubini theorem that mes $\Xi=\lambda_{0}$. For $\lambda \in \Xi$ the set of parameter values $a$ such that $F_{a, \lambda}$ admits an attractor with an absolutely continuous Bernoulli measure differs from $\mathfrak{M}$ by a set of measure 0 .

Proof of lemma 4. (1) First we fix $a$ and $\lambda$. For any $\tilde{y} \in \sigma$ there exists some $\tilde{z} \in \sigma$ such that

$$
\tilde{y}=\left(y_{0}, y_{1}, \ldots\right) \neq \tilde{z}=\left(z_{0}, z_{1}, \ldots\right)
$$

but $\pi(\tilde{y})=\pi(\tilde{z})=M \in \Lambda$. Let

$$
\begin{aligned}
& \sigma_{n}=\left\{y: \text { for some } \tilde{z} \pi \tilde{y}=\pi \tilde{z} \text { and } y_{i}=z_{i}\right. \\
& \left.\qquad \text { for } i=0,1, \ldots, n-1, \text { but } y_{n} \neq z_{n}\right\} .
\end{aligned}
$$


Consider the points

$$
\begin{gathered}
\tilde{y}^{-n}=\left(y_{n}, y_{n+1} \ldots\right), \quad \tilde{z}^{-n}=\left(z_{n}, z_{n+1} \cdots\right) \in \tilde{X}, \\
M_{1}^{-n}=\pi\left(\tilde{y}^{-n}\right), \quad M_{2}^{n}=\pi\left(\tilde{z}^{-n}\right) \in \Lambda .
\end{gathered}
$$

Then we have $M_{1}^{-n} \in \xi_{y_{n}}, M_{2}^{-n} \in \xi_{z_{n}} \rightarrow M_{k}^{-n} \neq M_{2}^{-n}$, but $\tau\left(M_{1}^{-n}\right)=\tau\left(M_{2}^{-n}\right)$. Let $\sigma_{n, k}$ be a subset of $\sigma_{n}$ defined by $y_{n} \in \Delta_{k \pm}$ (the corresponding $z_{n} \in \Delta_{k \mp}$ ). Let $\tilde{y}_{*}=\lim _{m \rightarrow \infty} \tilde{y}_{m}$ for a sequence $\tilde{y}_{m} \in \sigma_{n, k}$, and let $y_{* n}=\lim _{m \rightarrow \infty} y_{m n}$ be the $n$th coordinate of $\tilde{y}_{*}$. For $\tilde{z}_{m} \in \sigma_{n, k}$ satisfying $\pi\left(\tilde{z}_{m}\right)=\pi\left(\tilde{y}_{m}\right)$ consider some limit point $\tilde{z}_{*}$. As dist $\left(z_{n m}, y_{n m}\right)>c$ for any $m$ and $\tau\left(z_{n m}\right)=\tau\left(y_{n m}\right)$ we obtain using the continuity of $\tau$ that $z_{* n}=$ $\lim _{m \rightarrow \infty} z_{n m}$ is different from $y_{* n}$ and $\tau\left(z_{* n}\right)=\tau\left(y_{* n}\right)$. Hence $y_{*} \in \sigma_{n k}, \sigma_{n k}$ is closed, and $\sigma=\bigcup_{n, k} \sigma_{n k}$ is measurable.

It follows from the definition that $\tilde{G} \sigma_{n}=\sigma_{n+1}$. Thus $\sigma$ is invariant. As $\tilde{\mu}$ is ergodic $\tilde{\mu}(\sigma)=0$ or $\tilde{\mu}(\sigma)=\tilde{\mu}(\tilde{X})$ which proves (i).

(2) Now $a$ is fixed but $\lambda$ varies in $\left(0, \lambda_{0}\right]$. Then the sets $\sigma, \sigma_{n}, \sigma_{n k}$ depend on $\lambda$. Let $\mathfrak{B}$ be the set of $\lambda$ such that $\tilde{\mu}\left(\sigma_{\lambda}\right)=\tilde{\mu}(\tilde{X})$. As $\tilde{\mu}\left(\sigma_{\lambda n}\right)=\tilde{\mu}\left(\sigma_{\lambda n+1}\right)$ for $\lambda \in \mathfrak{B}$ we have $\tilde{\mu}\left(\sigma_{\lambda 1}\right)>0$. Let us define

$$
\mathfrak{B}_{m k}=\left\{\lambda \in \mathfrak{B}: \tilde{\mu}\left(\sigma_{\lambda 1 k}\right) \geq 1 / m\right\} .
$$

Then $\mathfrak{B}=\bigcup_{m, k} \mathfrak{B}_{m k}$. Suppose $\lambda_{*}=\lim _{n \rightarrow \infty} \lambda_{n}$ where $\lambda_{n} \in \mathfrak{B}_{m k}$ but $\lambda_{*} \notin \mathfrak{B}_{m k}$. Then

$$
\tilde{\mu}\left(\sigma_{\lambda_{* 1 k}}\right)=c_{*} \text { for some } 0 \leq c_{*}<1 / m \text {. }
$$

Let $d_{k \pm}=\left\{\tilde{x}=\left(x_{0}, x_{1}, x_{2} \cdots\right): x_{1} \in \Delta_{k \pm}\right\}$. If $\tilde{x} \in d_{k \pm} \mid \sigma_{1 k}$ then

$$
\operatorname{dist}\left[\tau\left(\pi\left(x_{1}, x_{2} \cdots\right), \tau \Delta_{k \mp}\right]>0\right. \text {. }
$$

Let

$$
V_{p}=\left\{\tilde{x}: \operatorname{dist}\left(\tau \pi\left(x_{1}, x_{2}, \ldots\right), \tau \Delta_{k \mp}\right) \geq 1 / p\right\} .
$$

Then $V_{p}$ is closed and

$$
\tilde{\mu}\left(\bigcup_{p} V_{p}\right)=\mu\left(\Delta_{k}\right)=c_{*}>\mu\left(\Delta_{k}\right)-1 / m
$$

For any $\varepsilon$ we can choose $P_{0}$ such that

$$
\tilde{\mu}\left(\bigcup_{p \leq P_{0}} V_{p}\right)>\mu\left(\Delta_{k}\right)-c_{*}-\varepsilon
$$

Using the continuous dependence of $\pi$ and $\tau$ from $\lambda$ we can choose $t>0$ such that any $\lambda \in\left(\lambda_{*}-t, \lambda_{*}+t\right)$ satisfies

$$
\tilde{\mu}\left(\sigma_{\lambda 1 k}\right)<\mu\left(\Delta_{k}\right)-\left(\mu\left(\Delta_{k}\right)-c_{*}-\varepsilon\right)<c_{*}+\varepsilon .
$$

For $\varepsilon$ sufficiently small and $\lambda=\lambda_{n}$ this contradicts to $\tilde{\mu}\left(\sigma_{\lambda_{n} 1 k}\right) \geq 1 / m$. Hence the sets $\mathfrak{B}_{m k}$ are closed and $\mathfrak{B}$ is measurable.

(3) Let mes $\mathfrak{B}>0$. Then for some $m, k$ mes $\mathfrak{B}_{m k}>0$. For $\tilde{x} \in \sigma_{\lambda 1 k+}$ we define

$$
\Gamma_{\tilde{x}}=\left\{\lambda: \pi_{\lambda}(\tilde{x}) \in \pi_{\lambda} \sigma_{\lambda 1 k-}\right\} \text {. }
$$

Applying the Fubini theorem to the product of the measure spaces

$$
(\tilde{X}, \tilde{\mu}) \times\left(\left[0, \lambda_{0}\right], d \lambda\right)
$$

we see that $\tilde{\mu}\left\{\tilde{x} \in \sigma_{\lambda 1 k+}\right.$ : mes $\left.\tilde{\Gamma}_{\tilde{x}}>0\right\}>0$. We fix some $\tilde{p}=\left(p_{0}, p_{1+}, p_{2}, \ldots\right)$ such that mes $\Gamma_{\tilde{p}}>0$. Then to any $\lambda \in \Gamma_{\tilde{p}}$ there corresponds some $\tilde{x}$ satisfying $\tilde{x}=$ 
$\left(x_{0}=p_{0}, x_{1}=p_{1-}, x_{2}, \ldots\right), \pi_{\lambda}(\tilde{x})=\pi_{\lambda}(\tilde{p})$. Now it is convenient to return to the inverse limit $([0, \tilde{1}], \tilde{g})$ of the initial one-dimensional map $g:[0,1] \rightarrow[0,1]$ and to the initial map $F:(x, y) \rightarrow\left(\varphi(y)+\lambda\left(x-\frac{1}{2}\right), g(y)\right)$ of the unit square. Let $q_{1+}=p_{1+}, q_{2}, q_{3}, \ldots$ be the coordinates of the point $\left(p_{1+}, p_{2}, p_{3}, \ldots\right) \in \tilde{X}$ considered as the point of $[\widetilde{0,1}]$. Set $q_{0}=g\left(q_{1+}\right)$ and let the point $\tilde{q}=\left(q_{0}, q_{1+}, q_{2}, \ldots\right) \in[\widetilde{0,1}]$ correspond to $\tilde{p} \in \tilde{X}$. Similarly to any $\tilde{x}=\left(p_{0}, p_{1-}, x_{2}, x_{3}, \ldots\right) \in \tilde{X}$ we associate $\tilde{y}=$ $\left(q_{0}, q_{1-}, y_{2}, y_{3}, \ldots\right) \in[\widetilde{0,1}]$. For uniformity we shall use $\Gamma_{\tilde{q}}$ instead of $\Gamma_{\tilde{p}}$. According to our assumption mes $\Gamma_{\tilde{q}}>0$ and for any $\lambda \in \Gamma_{\tilde{q}}$ there exists $\tilde{y}=\left(q_{0}, q_{1-}, y_{2}, \ldots\right)$ satisfying $\pi_{\lambda}(\tilde{y})=\pi_{\lambda}(\tilde{q})$.

(4) Applying subsequently formula (1) we obtain that if $\tilde{y}=\left(y_{0}, y_{1}, y_{2}, \ldots\right)$ then the second coordinate of the point $\pi_{\lambda}(\tilde{y})$ belonging to the unit square is $y_{0}$ and its first coordinate is given by

$$
s(\tilde{y}, \lambda)=\varphi\left(y_{1}\right)+\lambda\left(\varphi\left(y_{2}\right)-\frac{1}{2}\right)+\lambda^{2}\left(\varphi\left(y_{3}\right)-\frac{1}{2}\right)+\cdots
$$

If $\lambda_{1} \in \Gamma_{\tilde{q}}$ and $\tilde{y}=\tilde{y}\left(\lambda_{1}\right)=\left(q_{0}, q_{1-}, y_{2}, \ldots\right)$ is a point satisfying $\pi_{\lambda_{1}}(\tilde{y})=\pi_{\lambda_{1}}\left(\tilde{q}, \lambda_{1}\right)$ then $s\left(\tilde{y}, \lambda_{1}\right)=s\left(\tilde{q}, \lambda_{1}\right)$ but the series $s(\tilde{y}, \lambda)$ and $s(\tilde{q}, \lambda)$ do not coincide identically because their constant terms differ:

$$
\varphi\left(y_{1}\right)=\varphi\left(q_{1-}\right)=q_{1-}<\frac{1}{2}, \text { and } \varphi\left(q_{1+}\right)=q_{1+}>\frac{1}{2}
$$

(the map $F$ identifies points lying on $\xi_{q_{1-}}$ and on $\xi_{q_{1+}}$ only if $q_{1+}, q_{1-}$ are sufficiently close to $\frac{1}{2}$ and $\varphi(y) \equiv y$ for such points).

Let us denote by $s_{\lambda_{1}}(\tilde{y}, \delta \lambda)$ the series $s(\tilde{y}, \lambda)$ expressed as a power series in $\left(\lambda-\lambda_{1}\right)=\delta \lambda$. Then

$$
s_{\lambda_{1}}(\tilde{y}, \delta \lambda)=s_{0}\left(\tilde{y}, \lambda_{1}\right)+s_{1}\left(\tilde{y}, \lambda_{1}\right) \delta \lambda+\cdots+s_{n}\left(\tilde{y}, \lambda_{1}\right) \delta \lambda^{n}+\cdots .
$$

Since $s_{\lambda_{1}}(\tilde{y}, \delta \lambda) \neq s_{\lambda_{1}}(\tilde{q}, \delta \lambda)$ there is an $n$ such that $s_{n}\left(\tilde{y}, \lambda_{1}\right) \neq s_{n}\left(\tilde{q}, \lambda_{1}\right)$. For any rational $r>0$ let us define $\Gamma_{n, r} \subset \Gamma_{\tilde{q}}$ by

$$
\begin{gathered}
\Gamma_{n, r}=\left\{\lambda_{1}: \text { there exists a } \tilde{y} \text { satisfying } \pi_{\lambda_{1}}(\tilde{y})=\pi_{\lambda_{1}}(\tilde{q}), s_{i}\left(\tilde{y}, \lambda_{1}\right)=s_{i}\left(\tilde{q}, \lambda_{1}\right)\right. \\
\text { for } \left.i \in[1, n-1],\left|s_{n}\left(\tilde{q}, \lambda_{1}\right)-s_{n}\left(\tilde{y}, \lambda_{1}\right)\right| \geq r\right\} .
\end{gathered}
$$

One checks as above that $\Gamma_{n, r}$ are measurable. Thus mes $\Gamma_{n_{1}, r_{1}}>0$ for some choice of indices. Set $\Gamma=\Gamma_{n_{1}, r_{1}}$. Let $\Gamma_{1} \in \Gamma$ and let $\tilde{y}_{(1)}$ be the corresponding point satisfying $\pi_{\lambda_{1}}(\tilde{q})=\pi_{\lambda_{1}}\left(\tilde{y}_{(1)}\right)$. Then $s_{\lambda_{1}}(\tilde{q}, \delta \lambda), s_{\lambda_{1}}\left(\tilde{y}_{(1)}, \delta \lambda\right)$ may be written as

$$
\begin{gathered}
s_{\lambda_{1}}(\tilde{q}, \delta \lambda)=s_{0}\left(\lambda_{1}\right)+s_{1}\left(\lambda_{1}\right) \delta \lambda+\cdots+s_{n_{1}-1}\left(\lambda_{1}\right) \delta \lambda^{n_{1}-1}+s_{n_{1}}\left(\lambda_{1}\right) \delta \lambda^{n_{1}}+\cdots \\
s_{\lambda_{1}}\left(\tilde{y}_{(1)}, \delta \lambda\right)=s_{0}\left(\lambda_{1}\right)+s_{1}\left(\lambda_{1}\right) \delta \lambda+\cdots+s_{n_{1}-1}\left(\lambda_{1}\right) \delta \lambda^{n_{1}-1}+s_{n_{1}}\left(\tilde{y}_{(1)}, \lambda_{1}\right) \delta \lambda^{n_{1}}+\cdots
\end{gathered}
$$

Let us define

$$
\begin{gathered}
\theta(\delta \lambda)=s_{\lambda_{1}}(\tilde{q}, \delta \lambda)-s_{\lambda_{1}}\left(\tilde{y}_{(1)}, \delta \lambda\right), \\
\theta(\tilde{y}, \delta \lambda)=s_{\lambda_{1}}(\tilde{y}, \delta \lambda)-s_{\lambda_{1}}\left(\tilde{y}_{(1)}, \delta \lambda\right) .
\end{gathered}
$$

Then

$$
\begin{gathered}
(\delta \lambda)=\theta_{n_{1}}(\delta \lambda)^{n_{1}}+\theta_{n_{1}+1}(\delta \lambda)^{n_{1}+1}+\cdots \\
\theta(\tilde{y}, \delta \lambda)=\theta_{0}(\tilde{y})+\theta_{1}(\tilde{y}) \delta \lambda+\cdots+\theta_{n_{1}-1}(\tilde{y}) \delta \lambda^{n_{1}-1}+\theta_{n_{1}}(\tilde{y}) \delta \lambda^{n_{1}}+\cdots
\end{gathered}
$$

where $\left|\theta_{n_{1}}\right|>r_{1}$. 
(5) We fix some $\varepsilon_{1}, 0<\varepsilon_{1}<r_{1} / 2$. Let

$$
d\left(\tilde{y}, \tilde{y}^{\prime}\right)=\sum_{i=0}^{\infty}\left|y_{i}-y_{i}^{\prime}\right| / 2^{i}
$$

be the distance in $[\widetilde{0,1}]$. We denote by $B_{\varepsilon}(\tilde{y})$ the ball of radius $\varepsilon$ centred in $\tilde{y} \in[\widetilde{0,1}]$. There exists a $\delta_{1}=\delta_{1}\left(\varepsilon_{1}\right)>0$ satisfying the following conditions:

(a) if $\tilde{y} \in B_{\delta_{1}}\left(\tilde{y}_{(1)}\right)$ then $\left|\theta_{i}(\tilde{y})\right|<\varepsilon_{1 / 3}$ for $i \in\left[0, n_{1}\right]$;

(b) if $|\delta \lambda|<\delta_{1}$ then for any $\tilde{y}$

$$
\left|\frac{n_{1}+1}{2 !} \theta_{n_{1}+1}(\tilde{y}) \delta \lambda+\frac{\left(n_{1}+1\right)\left(n_{1}+2\right)}{3 !} \theta_{n_{1}+2}(\tilde{y}) \delta \lambda^{2}+\cdots\right|>\frac{\varepsilon_{1}}{3} .
$$

For $\lambda_{1} \in \Gamma$ we shall use $V\left(\lambda_{1}\right)$ to denote the non-empty set of $\tilde{y}=\left(q_{0}, q_{1-}, y_{2}, \ldots\right)$ appearing in the definition of $\lambda_{1}$, i.e.,

$$
\pi_{\Lambda_{1}}(\tilde{y})=\pi_{\lambda_{1}}(\tilde{q})
$$

the curves $s(\tilde{q}, \lambda), s(\tilde{y}, \lambda)$ have tangency of order $n_{1}-1$ at $\lambda=\lambda_{1}$, and

$$
\left|s_{n}\left(\tilde{q}, \lambda_{1}\right)-s_{n}\left(\tilde{y}, \lambda_{1}\right)\right|>r_{1} .
$$

Let

$$
\begin{aligned}
\Gamma\left(\lambda_{1}, \tilde{y}_{(1)}\right)=\left\{\delta \lambda:|\delta \lambda|<\delta_{1}, \lambda_{1}+\delta \lambda \in \Gamma,\right. \\
\\
\left.V\left(\lambda_{1}+\delta \lambda\right) \cap B_{\delta_{1}}\left(\tilde{y}_{(1)}\right) \neq \varnothing\right\} .
\end{aligned}
$$

Differentiating (27) $n_{1}-1$ times we obtain for

$$
\begin{gathered}
\delta \lambda \in \Gamma\left(\lambda_{1}, \tilde{y}_{(1)}\right) \text { and } \tilde{y} \in V\left(\lambda_{1}+\delta \lambda\right) \cap B_{\delta_{1}}\left(\tilde{y}_{(1)}\right) \\
\left(n_{1} !\right) \delta \lambda\left[\left(\theta_{n_{1}}-\theta_{n_{1}}(\tilde{y})\right)+\frac{n_{1}+1}{2 !}\left(\theta_{n_{1}+1}-\theta_{n_{1}+1}(\tilde{y})\right) \delta \lambda\right. \\
\left.+\frac{\left(n_{1}+1\right)\left(n_{1}+2\right)}{3 !}\left(\theta_{n_{1}+2}-\theta_{n_{1}+2}(\tilde{y})\right) \delta \lambda^{2}+\cdots\right]=\left(n_{1}-1\right) ! \theta_{n_{1}-1}(\tilde{y}) .
\end{gathered}
$$

Taking into account the choice of $\varepsilon_{1}$ and $\delta_{1}$ we obtain from (28)

$$
\delta \lambda(\tilde{y})=\frac{1}{n_{1}} \theta_{n_{1}-1}(\tilde{y}) \frac{1}{\theta_{n_{1}}(1+\varepsilon(\tilde{y}))}
$$

while $|\varepsilon(\tilde{y})|>\frac{1}{2}$.

(6) Let

$$
A_{k}\left(\lambda_{i}\right)=\left\{\left.\frac{d^{k}}{d \lambda^{k}} s(\tilde{y}, \lambda)\right|_{\lambda=\lambda_{1}}\right\} .
$$

One easily checks that for $\lambda_{1}<\frac{1}{2}$ mes $A_{k}\left(\lambda_{1}\right)=0$ for any $k$ (notice that $A_{0}\left(\lambda_{1}\right)=$ $\left.\left\{s\left(\tilde{y}, \lambda_{1}\right)\right\}=\Lambda \cap \xi_{q_{0}}\right\}$ ). When $\tilde{y}$ varies in $[\overline{0,1}]$ the values of $\theta_{n_{1}-1}(\tilde{y})$ belong to the set $A_{n_{1}-1}\left(\lambda_{1}\right)+c$ where

$$
c=\left.\frac{d^{n_{1}-1}}{d \lambda^{n_{1}-1}} s\left(\tilde{y}_{(1)}, \lambda\right)\right|_{\lambda=\lambda_{1}} .
$$

Using (29) we obtain from mes $A_{n_{1}-1}\left(\lambda_{1}\right)=0$ that mes $\Gamma\left(\lambda_{1}, \tilde{y}_{(1)}\right)=0$. 
Consider a cover $\left\{l_{i}\right\}_{i=1}^{m_{1}}$ of $\left[0, \lambda_{0}\right]$ by intervals of diam $<\delta_{1}$, and a cover $\left\{B_{\delta_{1} j}\right\}_{j=1}^{m_{1}}$ of the set $\tilde{y}=\left(q_{0}, q_{1-}, y_{2}, \ldots\right)$. Let

$$
\Gamma_{i j} \subset \Gamma=\left\{\lambda_{1} \in l_{i}: V\left(\lambda_{1}\right) \cap B_{j} \neq \varnothing\right\}
$$

The above arguments show that mes $\Gamma_{i j}=0$ for any choice of $i, j$. Hence mes $\Gamma=$ 0 , which proves lemma 4 .

\section{REFERENCES}

[1] V. M. Alexeyev, Quasi-random dynamical systems, I: Quasi-random diffeomorphisms. Math. of USSR-Sbornik 5 No. 1 (1968), 73-128.

[2] R. L. Adler, F-expansions revisited. Lecture Notes in Math. No. 318. Springer: Berlin, 1973, pp. 1-5.

[3] D. V. Anosov \& Ya. G. Sinai. Some smooth ergodic systems. Russian Math. Surveys 22 No. 5 (1967), 102-167.

[4] R. Bowen, On axiom A diffeomorphisms. Conference Board of the Mathematical Sciences. Regional Conference Series in Mathematics 35 (1978).

[5] F. Blanchard. $K$-flots et théorème de renouvellement. $Z$. Wahrscheinlichkeitstheorie verw. Gebiete 36 (1976), 345-358.

[6] B. M. Gurevich. Some existence conditions for $K$-decompositions for special flows. Tr. Mosc. Mat. Ob. 17 (1967), 89-116. (In Russian).

[7] J. Guckenheimer, G. Oster \& A. Ipaktchi. The dynamics of density dependent population models, Theor. Pop. Biol. 19 (1976).

[8] M. V. Jacobson. Absolutely continuous invariant measures for one-parameter families of onedimensioal maps. Commun. Math. Phys. 81 (1981), 39-88.

[9] M. V. Jacobson. Topological and metric properties of one-dimensional endomorphisms. Sov. Math. Dokl. 19 (1978), 1452-1455.

[10] F. Ledrappier. Some properties of absolutely continuous measures on an interval. Ergod. Th. \& Dynam. Sys. 1, No. 3 (1981).

[11] M. Ratner. Bernoulli flows over maps of the interval, Israel Math. J. 31 (1978), 298-314.

[12] V. A. Rohlin. Exact endomorphisms of a Lebesgue space. Izv. AN SSR, Ser. Mat. 25 (1961), 499-530. (In Russian).

[13] P. Walters. Invariant measures and equilibrium states for some mappings which expand distances. Trans. Amer. Math. Soc. 236 (1978), 121-153.

[14] R. Williams. One-dimensional non-wandering sets. Topology 6 (1967), 473-487. 